\title{
A WEAK DYNAMIC PROGRAMMING PRINCIPLE FOR COMBINED OPTIMAL STOPPING/STOCHASTIC CONTROL WITH $\mathcal{E}^{f}$-EXPECTATIONS*
}

\author{
ROXANA DUMITRESCU ${ }^{\dagger}$, MARIE-CLAIRE QUENEZ $^{\ddagger}$, AND AGNÈS SULEM ${ }^{\S}$
}

\begin{abstract}
We study a combined optimal control/stopping problem under a nonlinear expectation $\mathcal{E}^{f}$ induced by a BSDE with jumps, in a Markovian framework. The terminal reward function is only supposed to be Borelian. The value function $u$ associated with this problem is generally irregular. We first establish a sub- (resp., super-) optimality principle of dynamic programming involving its upper- (resp., lower-) semicontinuous envelope $u^{*}$ (resp., $u_{*}$ ). This result, called the weak dynamic programming principle (DPP), extends that obtained in [Bouchard and Touzi, SIAM J. Control Optim., 49 (2011), pp. 948-962] in the case of a classical expectation to the case of an $\mathcal{E}^{f}$-expectation and Borelian terminal reward function. Using this weak DPP, we then prove that $u^{*}$ (resp., $u_{*}$ ) is a viscosity sub- (resp., super-) solution of a nonlinear Hamilton-Jacobi-Bellman variational inequality.
\end{abstract}

Key words. Markovian stochastic control, mixed optimal control/stopping, nonlinear expectation, backward stochastic differential equation, weak dynamic programming principle, HamiltonJacobi-Bellman variational inequality, viscosity solution, $\mathcal{E}^{f}$-expectation

AMS subject classifications. 93E20, 60J60, 47N10

DOI. $10.1137 / 15 \mathrm{M} 1027012$

1. Introduction. Markovian stochastic control problems on a given horizon of time $T$ can typically be written as

$$
u(0, x)=\sup _{\alpha \in \mathcal{A}} \mathbb{E}\left[\int_{0}^{T} f\left(\alpha_{s}, X_{s}^{\alpha}\right) d s+g\left(X_{T}^{\alpha}\right)\right],
$$

where $\mathcal{A}$ is a set of admissible control processes $\alpha_{s}$, and $\left(X_{s}^{\alpha}\right)$ is a controlled process of the form

$$
X_{s}^{\alpha}=x+\int_{0}^{s} b\left(X_{u}^{\alpha}, \alpha_{u}\right) d u+\int_{0}^{s} \sigma\left(X_{u}^{\alpha}, \alpha_{u}\right) d W_{u}+\int_{0}^{s} \int_{\mathbb{R}^{n}} \beta\left(X_{u}^{\alpha}, \alpha_{u}, e\right) \tilde{N}(d u, d e) .
$$

The random variable $g\left(X_{T}^{\alpha}\right)$ may represent a terminal reward and $f\left(\alpha_{s}, X_{s}^{\alpha}\right)$ an instantaneous reward process. Formally, for all initial time $t$ in $[0, T]$ and initial state $y$, the associated value function is defined by

$$
u(t, y)=\sup _{\alpha \in \mathcal{A}} \mathbb{E}\left[\int_{t}^{T} f\left(\alpha_{s}, X_{s}^{\alpha}\right) d s+g\left(X_{T}^{\alpha}\right) \mid X_{t}^{\alpha}=y\right] .
$$

\footnotetext{
*Received by the editors June 19, 2015; accepted for publication (in revised form) May 17, 2016; published electronically August 24, 2016.

http://www.siam.org/journals/sicon/54-4/M102701.html

Funding: The research of the first author leading to these results has received funding from the Region Ile-de-France.

${ }^{\dagger}$ Institut für Mathematik, Humboldt-Universität zu Berlin, Unter den Linden 6, 10099 Berlin, Germany (roxana@ceremade.dauphine.fr).

${ }^{\ddagger}$ LPMA, Université Paris 7 Denis Diderot, Boite courrier 7012, 75251 Paris cedex 05, France (quenez@math.univ-paris-diderot.fr).

$\S$ INRIA Paris, 3 rue Simone Iff, CS 42112, 75589 Paris Cedex 12, France, and Université Paris-Est, Paris, France (agnes.sulem@inria.fr).
} 


\section{WEAK DPP WITH $\mathcal{E}^{f}$-EXPECTATIONS}

The dynamic programming principle (DPP) can formally be stated as

$$
u(0, x)=\sup _{\alpha \in \mathcal{A}} \mathbb{E}\left[\int_{0}^{t} f\left(\alpha_{s}, X_{s}^{\alpha}\right) d s+u\left(t, X_{t}^{\alpha}\right)\right] \text { for } t \text { in }[0, T] .
$$

This principle is classically established under assumptions which ensure that the value function $u$ satisfies some regularity properties. From this principle, it can be derived that the value function is a viscosity solution of the associated Hamilton-JacobiBellman (HJB) equation. Similar results are obtained for optimal stopping and mixed optimal stopping/control problems.

The case of a discontinuous value function and its links with viscosity solutions has been studied for deterministic control in the eighties. Barles and Perthame study in [3] a deterministic optimal stopping problem with a reward map $g$ only supposed to be Borelian. To this purpose, they introduce a notion of viscosity solution which extends the classical one to the discontinuous case: a function $v$ is said to be a weak viscosity solution of the HJB equation if its upper semicontinuous (u.s.c.) envelope, denoted by $v^{*}$, is a viscosity subsolution of this PDE, and if its the lower semicontinuous (l.s.c.) envelope, denoted by $v_{*}$, is a viscosity supersolution of this equation. Then, by the classical DPP provided in the previous literature, they get that the u.s.c. envelope $u^{*}$ of the value function satisfies a suboptimality principle in the sense of Lions and Souganidis in [19]. Using this suboptimality principle, they then show that $u^{*}$ is a viscosity subsolution of the HJB equation. Moreover, using the fact that the l.s.c. envelop $u_{*}$ of $u$ is the value function of a relaxed problem, they show that $u_{*}$ is a viscosity supersolution and thus get that $u$ is a weak viscosity solution of the HJB equation. They stress that in general, the weak viscosity solution of this PDE is not unique. However, under a regularity assumption on the reward $g$, by using the control formulae, they obtain that the u.s.c. envelope $u^{*}$ of the value function is the unique u.s.c. viscosity solution of the HJB equation. (See Remark 23 for additional references and comments.)

More recently, in a stochastic framework, Bouchard and Touzi [8] have proved a weak DPP when the terminal map $g$ is irregular: they prove that the value function $u$ satisfies a suboptimality principle of dynamic programming involving its u.s.c. envelope $u^{*}$, and under an additional regularity (lower semicontinuity) assumption of the reward $g$, they obtain a superoptimality principle involving the l.s.c. envelope $u_{*}$. Then, using the suboptimality principle, they derive that $u^{*}$ is a viscosity subsolution of the associated HJB equation. Moreover, when $g$ is l.s.c., using the superoptimality principle, they show that $u_{*}$ is a viscosity supersolution and thus get that $u$ is a weak viscosity solution of this PDE in the same sense as above (or [3]). A weak DPP has been further established, when $g$ is l.s.c. for problems with state constraints by Bouchard and Nutz in [7], and, when $g$ is continuous, for zero-sum stochastic games by Bayraktar and Yao in [4].

In this paper we are interested in generalizing these results to the case when $g$ is only Borelian and when the linear expectation $\mathbb{E}$ is replaced by a nonlinear expectation induced by a backward stochastic differential equation (BSDE) with jumps. Typically, such problems in the Markovian case can be formulated as

$$
\sup _{\alpha \in \mathcal{A}} \mathcal{E}_{0, T}^{\alpha}\left[g\left(X_{T}^{\alpha}\right)\right]
$$

where $\mathcal{E}^{\alpha}$ is the nonlinear expectation associated with a BSDE with jumps with controlled driver $f\left(\alpha_{t}, X_{t}^{\alpha}, y, z, k\right)$. Note that problem (1.1) is a particular case of 
(1.4) when the driver $f$ does not depend on the solution of the BSDE, that is, when $f\left(\alpha_{t}, X_{t}^{\alpha}, y, z, k\right) \equiv f\left(\alpha_{t}, X_{t}^{\alpha}\right)$.

We first provide a weak DPP involving the u.s.c. and l.s.c. envelopes of the value function. For this purpose, we prove some preliminary results, in particular some measurability and "splitting" properties. No regularity condition on $g$ is required to obtain the sub- and super-optimality principles, which is not the case in the previous literature in the stochastic case, even with a classical expectation (see [8], [7], and [4]). Using this weak DPP, we then show that the value function, which is generally neither u.s.c. nor l.s.c., is a weak viscosity solution (in the sense of [3]) of an associated nonlinear HJB equation.

Moreover, in this paper, we consider the combined problem when there is an additional control in the form of a stopping time. We thus consider mixed generalized optimal control/stopping problems of the form

$$
\sup _{\alpha \in \mathcal{A}} \sup _{\tau \in \mathcal{T}} \mathcal{E}_{0, \tau}^{\alpha}\left[\bar{h}\left(\tau, X_{\tau}^{\alpha}\right)\right]
$$

where $\mathcal{T}$ denotes the set of stopping times with values in $[0, T]$, and $\bar{h}$ is an irregular reward function.

Note that in the literature on BSDEs, some papers (see, e.g., Peng [20], Li and Peng [18], Buckdahn and Li [9], and Buckdahn and Nie [10]) study stochastic control problems with nonlinear $\mathcal{E}$-expectation in the continuous case (without optimal stopping). Their approach is different from ours and relies on the continuity assumption of the reward function.

The paper is organized as follows: in section 2, we formulate our generalized mixed control-optimal stopping problem. Using results on reflected BSDEs (RBSDEs), we express this problem as an optimal control problem for RBSDEs. In section 3, we prove a weak DPP for our mixed problem with $\mathcal{E}^{f}$-expectation. This requires some specific techniques of stochastic analysis and BSDEs to handle measurability and other issues due to the nonlinearity of the expectation and the lack of regularity of the terminal reward. Using the DPP and properties of RBSDEs, we prove in section 4 that the value function of our mixed problem is a weak viscosity solution of a nonlinear HJB variational inequality. In the appendix, we give several fine measurability properties which are used in the paper.

2. Formulation of the mixed stopping/control problem. We consider the product space $\Omega:=\Omega_{W} \otimes \Omega_{N}$, where $\Omega_{W}:=\mathcal{C}([0, T])$ is the Wiener space, that is, the set of continuous functions $\omega^{1}$ from $[0, T]$ into $\mathbb{R}^{p}$ such that $\omega^{1}(0)=0$, and $\Omega_{N}:=\mathbb{D}([0, T])$ is the Skorohod space of right-continuous with left limits (RCLL) functions $\omega^{2}$ from $[0, T]$ into $\mathbb{R}^{d}$, such that $\omega^{2}(0)=0$. Recall that $\Omega$ is a Polish space for the Skorohod metric. Here $p, d \geq 1$, but, for notational simplicity, we shall consider only $\mathbb{R}$-valued functions, that is, the case $p=d=1$.

Let $B=\left(B^{1}, B^{2}\right)$ be the canonical process defined for each $t \in[0, T]$ and each $\omega=\left(\omega^{1}, \omega^{2}\right)$ by $B_{t}^{i}(\omega)=B_{t}^{i}\left(\omega^{i}\right):=\omega_{t}^{i}$ for $i=1,2$. Let us denote the first coordinate process $B^{1}$ by $W$. Let $P^{W}$ be the probability measure on $\left(\Omega_{W}, \mathcal{B}\left(\Omega_{W}\right)\right)$ such that $W$ is a Brownian motion. Here $\mathcal{B}\left(\Omega_{W}\right)$ denotes the Borelian $\sigma$-algebra on $\Omega_{W}$.

Set $\mathbf{E}:=\mathbb{R}^{n} \backslash\{0\}$ equipped with its Borelian $\sigma$-algebra $\mathcal{B}(\mathbf{E})$, where $n \geq 1$. We define the jump random measure $N$ as follows: for each $t>0$ and each $\mathbf{B} \in \mathcal{B}(\mathbf{E})$,

$$
N(.,[0, t] \times \mathbf{B}):=\sum_{0<s \leq t} \mathbf{1}_{\left\{\Delta B_{s}^{2} \in \mathbf{B}\right\}} .
$$


The measurable set $(\mathbf{E}, \mathcal{B}(\mathbf{E}))$ is equipped with a $\sigma$-finite positive measure $\nu$ such that $\int_{\mathbf{E}}(1 \wedge|e|) \nu(d e)<\infty$. Let $P^{N}$ be the probability measure on $\left(\Omega_{N}, \mathcal{B}\left(\Omega_{N}\right)\right)$ such that $N$ is a Poisson random measure with compensator $\nu(d e) d t$ and such that $B_{t}^{2}=\sum_{0<s \leq t} \Delta B_{s}^{2}$ a.s. Note that the sum of jumps is well defined up to a $P^{N}$-null set. We set $\tilde{N}(d r, d e):=N(d r, d e)-\nu(d e) d t$. The space $\Omega$ is equipped with the $\sigma$-algebra $\mathcal{B}(\Omega)$ and the probability measure $P:=P^{W} \otimes P^{N}$. Let $\mathbb{F}:=\left(\mathcal{F}_{t}\right)_{t \geq 0}$ be the filtration generated by $W$ and $N$ completed with respect to $\mathcal{B}(\Omega)$ and $P$, defined as follows (see $[17$, p. 3$]$ or $[13, \mathrm{IV}]:$ let $\mathcal{F}$ be the completion $\sigma$-algebra of $\mathcal{B}(\Omega)$ with respect to $P^{1}$ For each $t \in[0, T], \mathcal{F}_{t}$ is the $\sigma$-algebra generated by $W_{s}, N_{s}, s \leq t$ and the $P$-null sets. Note that $\mathcal{F}_{T}=\mathcal{F}$ and $\mathcal{F}_{0}$ is the $\sigma$-algebra generated by the $P$-null sets. Let $\mathcal{P}$ be the predictable $\sigma$-algebra on $\Omega \times[0, T]$ associated with the filtration $\mathbb{F}$.

Let $T>0$ be fixed. Let $\mathbb{H}_{T}^{2}$ (denoted also by $\mathbb{H}^{2}$ ) be the set of real-valued predictable processes $\left(Z_{t}\right)$ such that $\mathbb{E} \int_{0}^{T} Z_{s}^{2} d s<\infty$ and let $\mathcal{S}^{2}$ be the set of real-valued RCLL adapted processes $\left(\varphi_{s}\right)$ with $\mathbb{E}\left[\sup _{0 \leq s \leq T} \varphi_{s}^{2}\right]<\infty$. Let $L_{\nu}^{2}$ be the set of measurable functions $l:(\mathbf{E}, \mathcal{B}(\mathbf{E})) \rightarrow(\mathbb{R}, \mathcal{B}(\mathbb{R}))$ such that $\|l\|_{\nu}^{2}:=\int_{\mathbf{E}} l^{2}(e) \nu(d e)<\infty$. The set $L_{\nu}^{2}$ is a Hilbert space equipped with the scalar product $\left\langle l, l^{\prime}\right\rangle_{\nu}:=\int_{\mathbf{E}} l(e) l^{\prime}(e) \nu(d e)$ for all $l, l^{\prime} \in L_{\nu}^{2} \times L_{\nu}^{2}$. Let $\mathbb{H}_{\nu}^{2}$ denote the set of predictable real-valued processes $\left(k_{t}(\cdot)\right)$ with $\mathbb{E} \int_{0}^{T}\left\|k_{s}\right\|_{L_{\nu}^{2}}^{2} d s<\infty$.

Let $\mathcal{A}$ be the set of controls, defined as the set of predictable processes $\alpha$ valued in a compact subset $\mathbf{A}$ of $\mathbb{R}^{p}$, where $p \in \mathbb{N}^{*}$. For each $\alpha \in \mathcal{A}$ and each initial condition $x$ in $\mathbb{R}$, let $\left(X_{s}^{\alpha, x}\right)_{0 \leq s \leq T}$ be the unique $\mathbb{R}$-valued solution in $\mathcal{S}^{2}$ of the stochastic differential equation (SDE):

$X_{s}^{\alpha, x}=x+\int_{0}^{s} b\left(X_{r}^{\alpha, x}, \alpha_{r}\right) d r+\int_{0}^{s} \sigma\left(X_{r}^{\alpha, x}, \alpha_{r}\right) d W_{r}+\int_{0}^{s} \int_{\mathbf{E}} \beta\left(X_{r^{-}}^{\alpha, x}, \alpha_{r}, e\right) \tilde{N}(d r, d e)$,

where $b, \sigma: \mathbb{R} \times \mathbf{A} \rightarrow \mathbb{R}$, are Lipschitz continuous with respect to $x$ and $\alpha$, and $\beta: \mathbb{R} \times \mathbf{A} \times \mathbf{E} \rightarrow \mathbb{R}$ is a bounded measurable function such that for some constant $C \geq 0$, and for all $e \in \mathbf{E}$

$$
\begin{aligned}
|\beta(x, \alpha, e)| & \leq C \Psi(e), \quad x \in \mathbb{R}, \alpha \in \mathbf{A}, \quad \text { where } \quad \Psi \in L_{\nu}^{2}, \\
\left|\beta(x, \alpha, e)-\beta\left(x^{\prime}, \alpha^{\prime}, e\right)\right| & \leq C\left(\left|x-x^{\prime}\right|+\left|\alpha-\alpha^{\prime}\right|\right) \Psi(e), \quad x, x^{\prime} \in \mathbb{R}, \alpha, \alpha^{\prime} \in \mathbf{A} .
\end{aligned}
$$

The criterion of our mixed control problem, depending on $\alpha$, is defined via a BSDE with driver function $f$ satisfying the following hypothesis.

Assumption 1. $f: \mathbf{A} \times[0, T] \times \mathbb{R}^{3} \times L_{\nu}^{2} \rightarrow(\mathbb{R}, \mathcal{B}(\mathbb{R}))$ is $\mathcal{B}(\mathbf{A}) \otimes \mathcal{B}([0, T]) \otimes \mathcal{B}\left(\mathbb{R}^{3}\right) \otimes$ $\mathcal{B}\left(L_{\nu}^{2}\right)$-measurable and satisfies

(i) $|f(\alpha, t, x, 0,0,0)| \leq C\left(1+|x|^{p}\right)$ for all $\alpha \in \mathbf{A}, t \in[0, T], x \in \mathbb{R}$, where $p \in \mathbb{N}^{*}$,

(ii) $\left|f(\alpha, t, x, y, z, k)-f\left(\alpha^{\prime}, t, x^{\prime}, y^{\prime}, z^{\prime}, k^{\prime}\right)\right| \leq C\left(\left|\alpha-\alpha^{\prime}\right|+\left|x-x^{\prime}\right|+\left|y-y^{\prime}\right|+\right.$ $\left.\left|z-z^{\prime}\right|+\left\|k-k^{\prime}\right\|_{L_{\nu}^{2}}\right)$ for all $t \in[0, T], x, x^{\prime}, y, y^{\prime}, z, z^{\prime} \in \mathbb{R}, k, k^{\prime} \in L_{\nu}^{2}, \alpha, \alpha^{\prime} \in \mathbf{A}$,

(iii) $f\left(\alpha, t, x, y, z, k_{2}\right)-f\left(\alpha, t, x, y, z, k_{1}\right) \geq\left\langle\gamma\left(\alpha, t, x, y, z, k_{1}, k_{2}\right), k_{2}-k_{1}\right\rangle_{\nu}$ for all $t, x, y, z, k_{1}, k_{2}, \alpha$

where $\gamma: \mathbf{A} \times[0, T] \times \mathbb{R}^{3} \times\left(L_{\nu}^{2}\right)^{2} \rightarrow\left(L_{\nu}^{2}, \mathcal{B}\left(L_{\nu}^{2}\right)\right)$ is $\mathcal{B}(\mathbf{A}) \otimes \mathcal{B}([0, T]) \otimes \mathcal{B}\left(\mathbb{R}^{3}\right) \otimes \mathcal{B}\left(\left(L_{\nu}^{2}\right)^{2}\right)$ measurable, satisfying $\gamma().(e) \geq-1$ and $|\gamma().(e)| \leq \Psi(e), d \nu(e)$-a.s., where $\Psi \in L_{\nu}^{2}$.

For all $x \in \mathbb{R}$ and all control $\alpha \in \mathcal{A}$, let $f^{\alpha, x}$ be the driver defined by

$$
f^{\alpha, x}(r, \omega, y, z, k):=f\left(\alpha_{r}(\omega), r, X_{r}^{\alpha, x}(\omega), y, z, k\right) .
$$

\footnotetext{
${ }^{1}$ For the definition of the completion of a $\sigma$-algebra and the one of $P$-null sets, see, e.g., Lemma 26.
} 
We introduce the nonlinear expectation $\mathcal{E}^{f^{\alpha, x}}$ (denoted more simply by $\mathcal{E}^{\alpha, x}$ ) associated with $f^{\alpha, x}$, defined for each stopping time $\tau$ and for each $\eta \in L^{2}\left(\mathcal{F}_{\tau}\right)$ as

$$
\mathcal{E}_{r, \tau}^{\alpha, x}[\eta]:=\mathcal{X}_{r}^{\alpha, x}, \quad 0 \leq r \leq \tau,
$$

where $\left(\mathcal{X}_{r}^{\alpha, x}\right)$ is the solution in $\mathcal{S}^{2}$ of the BSDE associated with driver $f^{\alpha, x}$, terminal time $\tau$, and terminal condition $\eta$, that is satisfying

$$
\begin{aligned}
-d \mathcal{X}_{r}^{\alpha, x}= & f\left(\alpha_{r}, r, X_{r}^{\alpha, x}, \mathcal{X}_{r}^{\alpha, x}, Z_{r}^{\alpha, x}, K_{r}^{\alpha, x}(\cdot)\right) d r \\
& -Z_{r}^{\alpha, x} d W_{r}-\int_{\mathbf{E}} K_{r}^{\alpha, x}(e) \tilde{N}(d r, d e) ; \quad \mathcal{X}_{S}^{\alpha, x}=\eta,
\end{aligned}
$$

and $\left(Z_{s}^{\alpha, x}\right),\left(K_{s}^{\alpha, x}\right)$ are the associated processes, which belong respectively to $\mathbb{H}^{2}$ and $\mathbb{H}_{\nu}^{2}$. Condition (iii) ensures the nondecreasing property of the $\mathcal{E}^{f^{\alpha, x}}$-expectation (see [21]).

For all $x \in \mathbb{R}$ and all control $\alpha \in \mathcal{A}$, we define the reward by $h\left(s, X_{s}^{\alpha, x}\right)$ for $0 \leq s<T$ and $g\left(X_{T}^{\alpha, x}\right)$ for $s=T$, where

- $g: \mathbb{R} \rightarrow \mathbb{R}$ is Borelian,

- $h:[0, T] \times \mathbb{R} \rightarrow \mathbb{R}$ is a function which is Lipschitz continuous with respect to $x$ uniformly in $t$, and continuous with respect to $t$ on $[0, T]$,

- $|h(t, x)|+|g(x)| \leq C\left(1+|x|^{p}\right)$ for all $t \in[0, T], x \in \mathbb{R}$, with $p \in \mathbb{N}^{*}$.

Let $\mathcal{T}$ be the set of stopping times with values in $[0, T]$. Suppose the initial time is equal to 0 . Note that $\mathcal{E}_{0, \tau}^{\alpha, t, x}\left[\bar{h}\left(\tau, X_{\tau}^{\alpha, t, x}\right)\right]$ can be taken as constant. ${ }^{2}$ For each initial condition $x \in \mathbb{R}$, we consider the mixed optimal control/stopping problem:

$$
u(0, x):=\sup _{\alpha \in \mathcal{A}} \sup _{\tau \in \mathcal{T}} \mathcal{E}_{0, \tau}^{\alpha, x}\left[\bar{h}\left(\tau, X_{\tau}^{\alpha, x}\right)\right],
$$

where

$$
\bar{h}(t, x):=h(t, x) \mathbf{1}_{t<T}+g(x) \mathbf{1}_{t=T} .
$$

Note that $\bar{h}$ is Borelian but not necessarily regular in $(t, x)$.

We now make the problem dynamic. We define for $t \in[0, T]$ and each $\omega \in \Omega$ the $t$-translated path $\omega^{t}=\left(\omega_{s}^{t}\right)_{s \geq t}:=\left(\omega_{s}-\omega_{t}\right)_{s \geq t}$. Note that $\left(\omega_{s}^{1, t}\right)_{s \geq t}:=\left(\omega_{s}^{1}-\omega_{t}^{1}\right)_{s \geq t}$ corresponds to the realizations of the translated Brownian motion $W^{t}:=\left(W_{s}-W_{t}\right)_{s \geq t}$ and that the translated Poisson random measure $\left.\left.N^{t}:=N(] t, s\right],\right)_{s \geq t}$ can be expressed in terms of $\left(\omega_{s}^{2, t}\right)_{s \geq t}:=\left(\omega_{s}^{2}-\omega_{t}^{2}\right)_{s \geq t}$ similarly to $(2.1)$. Let $\mathbb{F}^{t}=\left(\mathcal{F}_{s}^{t}\right)_{t \leq s \leq T}$ be the filtration generated by $W^{t}$ and $N^{t}$ completed with respect to $\mathcal{B}(\Omega)$ and $P$. Note that for each $s \in[t, T], \mathcal{F}_{s}^{t}$ is the $\sigma$-algebra generated by $W_{r}^{t}, N_{r}^{t}, t \leq r \leq s$, and $\mathcal{F}_{0}$. Recall also that we have a martingale representation theorem for $\mathbb{F}^{t}$-martingales as stochastic integrals with respect to $W^{t}$ and $\tilde{N}^{t}$.

Let us denote by $\mathcal{T}_{t}^{t}$ the set of stopping times with respect to $\mathbb{F}^{t}$ with values in $[t, T]$. Let $\mathcal{P}^{t}$ be the predictable $\sigma$-algebra on $\Omega \times[t, T]$ equipped with the filtration $\mathbb{F}^{t}$.

We now introduce the following spaces of processes. Let $t \in[0, T]$. Let $\mathbb{H}_{t}^{2}$ be the $\mathcal{P}^{t}$-measurable processes $Z$ on $\Omega \times[t, T]$ such that $\|Z\|_{\mathbb{H}_{t}^{2}}:=\mathbb{E}\left[\int_{t}^{T} Z_{u}^{2} d u\right]<\infty$. We define $\mathbb{H}_{t, \nu}^{2}$ as the set of $\mathcal{P}^{t}$-measurable processes $K$ on $\Omega \times[t, T]$ such that $\|K\|_{\mathbb{H}_{t, \nu}^{2}}:=$ $\mathbb{E}\left[\int_{t}^{T}\left\|K_{u}\right\|_{\nu}^{2} d u\right]<\infty$. We denote by $\mathcal{S}_{t}^{2}$ the set of real-valued RCLL processes $\varphi$ on $\Omega \times[t, T], \mathbb{F}^{t}$-adapted, with $\mathbb{E}\left[\sup _{t \leq s \leq T} \varphi_{s}^{2}\right]<\infty$.

\footnotetext{
${ }^{2}$ Indeed, the solution of a BSDE with a Lipschitz driver is unique up to a $P$-null set. Its initial value may thus be taken constant for all $\omega$, modulo a change of its value on a $P$-null set, because $\mathcal{F}_{0}$ is the $\sigma$-algebra generated by the $P$-null sets.
} 
Let $\mathcal{A}_{t}^{t}$ be the set of controls $\alpha: \Omega \times[t, T] \mapsto \mathbf{A}$, which are $\mathcal{P}^{t}$-measurable. We consider the solution denoted by $X^{\alpha, t, x}$ in $\mathcal{S}_{t}^{2}$ of the following SDE driven by the translated Brownian motion $W^{t}$ and the translated Poisson random measure $N^{t}$ $\left(\right.$ with filtration $\left.\mathbb{F}^{t}\right)$ :

$$
\begin{aligned}
X_{s}^{\alpha, t, x}= & x+\int_{t}^{s} b\left(X_{r}^{\alpha, t, x}, \alpha_{r}\right) d r+\int_{t}^{s} \sigma\left(X_{r}^{\alpha, t, x}, \alpha_{r}\right) d W_{r}^{t} \\
& +\int_{t}^{s} \int_{\mathbf{E}} \beta\left(X_{r^{-}}^{\alpha, t, x}, \alpha_{r}, e\right) \tilde{N}^{t}(d r, d e) .
\end{aligned}
$$

For all $(t, x) \in[0, T] \times \mathbb{R}$ and all control $\alpha \in \mathcal{A}_{t}^{t}$, let $f^{\alpha, t, x}$ be the driver defined by

$$
f^{\alpha, t, x}(r, \omega, y, z, k):=f\left(\alpha_{r}(\omega), r, X_{r}^{\alpha, t, x}(\omega), y, z, k\right) .
$$

Let $\mathcal{E}_{., \tau}^{\alpha, t, x}\left[\bar{h}\left(\tau, X_{\tau}^{\alpha, t, x}\right)\right]$ (denoted also by $\mathcal{X}^{\alpha, t, x}$ ) be the solution in $\mathcal{S}_{t}^{2}$ of the BSDE with driver $f^{\alpha, t, x}$, terminal time $\tau$, and terminal condition $\bar{h}\left(\tau, X_{\tau}^{\alpha, t, x}\right)$, driven by $W^{t}$ and $N^{t}$, which is solved on $[t, T] \times \Omega$ with respect to the filtration $\mathbb{F}^{t}$ :

$$
\left\{\begin{array}{c}
-d \mathcal{X}_{r}^{\alpha, t, x}=f\left(\alpha_{r}, r, X_{r}^{\alpha, t, x}, \mathcal{X}_{r}^{\alpha, t, x}, Z_{r}^{\alpha, t, x}, K_{r}^{\alpha, t, x}\right) d r \\
-Z_{r}^{\alpha, t, x} d W_{r}^{t}-\int_{\mathbf{E}} K_{r}^{\alpha, t, x}(e) \tilde{N}^{t}(d r, d e) \\
\mathcal{X}_{\tau}^{\alpha, t, x}=\bar{h}\left(\tau, X_{\tau}^{\alpha, t, x}\right),
\end{array}\right.
$$

where $Z^{\alpha, t, x}, K^{\alpha, t, x}$ are the associated processes, which belong, respectively, to $\mathbb{H}_{t}^{2}$ and $\mathbb{H}_{t, \nu}^{2}$. Note that $\mathcal{E}_{t, \tau}^{\alpha, t, x}\left[\bar{h}\left(\tau, X_{\tau}^{\alpha, t, x}\right)\right]$ can be taken deterministic modulo a change of its value on a $P$-null set. ${ }^{3}$

For each initial time $t$ and each initial condition $x$, we define the value function as

$$
u(t, x):=\sup _{\alpha \in \mathcal{A}_{t}^{t}} \sup _{\tau \in \mathcal{T}_{t}^{t}} \mathcal{E}_{t, \tau}^{\alpha, t, x}\left[\bar{h}\left(\tau, X_{\tau}^{\alpha, t, x}\right)\right]
$$

which is a deterministic function of $t$ and $x$.

For each $\alpha \in \mathcal{A}_{t}^{t}$, we introduce the function $u^{\alpha}$ defined as

$$
u^{\alpha}(t, x):=\sup _{\tau \in \mathcal{T}_{t}^{t}} \mathcal{E}_{t, \tau}^{\alpha, t, x}\left[\bar{h}\left(\tau, X_{\tau}^{\alpha, t, x}\right)\right]
$$

We thus get

$$
u(t, x)=\sup _{\alpha \in \mathcal{A}_{t}^{t}} u^{\alpha}(t, x) .
$$

For each $\alpha, u^{\alpha}(t, x) \geq \bar{h}(t, x)$, and hence $u(t, x) \geq \bar{h}(t, x)$. Moreover, $u^{\alpha}(T, x)=$ $u(T, x)=g(x)$.

By [22, Theorem 3.2], for each $\alpha$, the value function $u^{\alpha}$ is related to a reflected BSDE. More precisely, let $\left(Y^{\alpha, t, x}, Z^{\alpha, t, x}, K^{\alpha, t, x}\right) \in \mathcal{S}_{t}^{2} \times \mathbb{H}_{t}^{2} \times \mathbb{H}_{\nu, t}^{2}$ be the solution of the reflected BSDE associated with driver $f^{\alpha, t, x}:=f\left(\alpha ., \cdot, X^{\alpha, t, x}, y, z, k\right)$, RCLL

\footnotetext{
${ }^{3}$ Indeed, the solution $\mathcal{E}_{., \tau}^{\alpha, t, x}\left[\bar{h}\left(\tau, X_{\tau}^{\alpha, t, x}\right)\right]\left(=\mathcal{X}^{\alpha, t, x}\right)$ of the BSDE (2.5) is unique up to a $P$-null set. Moreover, its value at time $t$ is $\mathcal{F}_{t}^{t}$-measurable, and $\mathcal{F}_{t}^{t}$ is equal to the $\sigma$-algebra generated by the $P$-null sets (that is, $\mathcal{F}_{0}$ ). The same property holds for the solution of the reflected BSDE (2.8). See also the additional Remarks 6 and 8 .
} 
obstacle process $\xi_{s}^{\alpha, t, x}:=\bar{h}\left(s, X_{s}^{\alpha, t, x}\right)_{t \leq s \leq T}$, terminal condition $g\left(X_{T}^{\alpha, t, x}\right)$, and with filtration $\mathbb{F}^{t}$, that is,

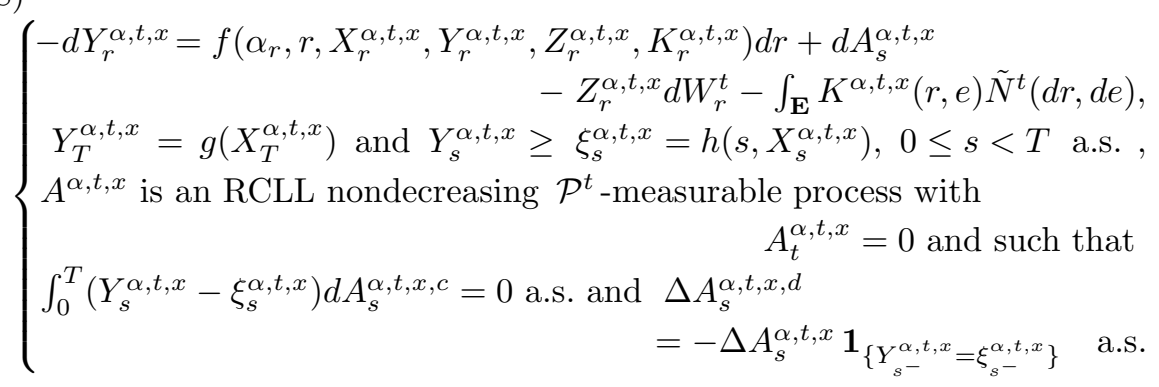

Here $A^{\alpha, t, x, c}$ denotes the continuous part of $A$ and $A^{\alpha, t, x, d}$ its discontinuous part. In the particular case when $h(T, x) \leq g(x)$, the obstacle $\xi^{\alpha, t, x}$ satisfies for all $\mathbb{F}^{t}$ predictable stopping time $\tau, \xi_{\tau^{-}} \leq \xi_{\tau}$ a.s., which implies the continuity of the process $A^{\alpha, t, x}$ (see [22]).

In the following, for each $\alpha \in \mathcal{A}_{t}^{t}, Y_{.}^{\alpha, t, x}$ will be also denoted by $Y_{,, T}^{\alpha, t, x}\left[g\left(X_{T}^{\alpha, t, x}\right)\right]$. Note that its value at time $t$ can be taken as deterministic modulo a change of its value on a $P$-null set.

Using [22, Theorem 3.2], we get that for each $\alpha \in \mathcal{A}_{t}^{t}$,

$$
u^{\alpha}(t, x)=Y_{t}^{\alpha, t, x}=Y_{t, T}^{\alpha, t, x}\left[g\left(X_{T}^{\alpha, t, x}\right)\right] .
$$

By using these equalities, we can reduce our mixed optimal stopping/control problem (2.6) to an optimal control problem for reflected BSDEs.

TheOREm 2 (characterization of the value function). For each $(t, x) \in[0, T] \times \mathbb{R}$, the value function $u(t, x)$ of the mixed optimal stopping/ control problem (2.6) satisfies

$$
u(t, x)=\sup _{\alpha \in \mathcal{A}_{t}^{t}} u^{\alpha}(t, x)=\sup _{\alpha \in \mathcal{A}_{t}^{t}} Y_{t, T}^{\alpha, t, x}\left[g\left(X_{T}^{\alpha, t, x}\right)\right] .
$$

This key property will be used to solve our mixed problem. We point out that in the classical case of linear expectations, this approach allows us to provide alternative proofs of the DPP to those given in the previous literature.

Remark 3. Some mixed optimal control/stopping problems with nonlinear expectations have been studied in [5, 22]. In these papers, the reward process does not depend on the control, which yields the characterization of the value function as the solution of an RBSDE. This is not the case here.

3. Weak DPP. In this section, we prove a weak DPP for our mixed optimal control/stopping problem (2.6). To this purpose, we first provide some splitting properties for the forward-backward system $(2.4)-(2.8)$. We then show some measurability properties of the function $u^{\alpha}(t, x)$, defined by (2.9), with respect to both the state variable $x$ and the control $\alpha$. Using these results, we show the existence of $\varepsilon$-optimal controls satisfying some appropriate measurability properties. Moreover, we establish a Fatou lemma for RBSDEs, where the limit involves both terminal condition and terminal time. Using these results, we then prove a sub- (resp., super-) optimality principle of dynamic programming, involving the u.s.c. (resp., l.s.c.) envelope of the value function. 
3.1. Splitting properties. Let $s \in[0, T]$. For each $\omega$, let ${ }^{s} \omega:=\left(\omega_{r \wedge s}\right)_{0 \leq r \leq T}$ and $\omega^{s}:=\left(\omega_{r}-\omega_{s}\right)_{s \leq r \leq T}$. We shall identify the path $\omega$ with $\left({ }^{s} \omega, \omega^{s}\right)$, which means that a path can be split into two parts: the path before time $s$ and the $s$-translated path after time $s$.

Let $\alpha$ be a given control in $\mathcal{A}$. We show below the following: at time $s$, for fixed past path $\tilde{\omega}:={ }^{s} \omega$, the process $\alpha(\tilde{\omega},$.$) which only depends on the future path \omega^{s}$ is an $s$-admissible control, that is, $\alpha(\tilde{\omega},.) \in \mathcal{A}_{s}^{s}$; furthermore, the criterium $Y^{\alpha, 0, x}(\tilde{\omega},$. from time $s$ coincides with the solution of the reflected BSDE driven by $W^{s}$ and $\tilde{N}^{s}$, controlled by $\alpha(\tilde{\omega},$.$) and associated with initial time s$ and initial state condition $X_{s}^{\alpha, 0, x}(\tilde{\omega})$.

We introduce the following random variables defined on $\Omega$ by

$$
S^{s}: \omega \mapsto{ }^{s} \omega ; \quad T^{s}: \omega \mapsto \omega^{s} .
$$

Note that they are independent. For each $\omega \in \Omega$, we have $\omega=S^{s}(\omega)+T^{s}(\omega) \mathbf{1}_{] s, T]}$, or equivalently $\omega_{r}=\omega_{r \wedge s}+\omega_{r}^{s} \mathbf{1}_{s, T]}(r)$, for all $r \in[0, T]$.

For all paths $\omega, \omega^{\prime} \in \Omega,\left({ }^{s} \omega, T^{s}\left(\omega^{\prime}\right)\right)$ denotes the path such that the past trajectory before $s$ is that of $\omega$, and the $s$-translated trajectory after $s$ is that of $\omega^{\prime}$. This can also be written as $\left({ }^{s} \omega, T^{s}\left(\omega^{\prime}\right)\right):={ }^{s} \omega+T^{s}\left(\omega^{\prime}\right) \mathbf{1}_{] s, T]}$. Note that for each $\omega \in \Omega$, we have $\left({ }^{s} \omega, T^{s}(\omega)\right)=\omega$.

Lemma 4. Let $s \in[0, T]$. Let $Z \in \mathbb{H}^{2}$. There exists a $P$-null set $\mathcal{N}$ such that for each $\omega$ in the complement $\mathcal{N}^{c}$ of $\mathcal{N}$, setting $\tilde{\omega}:={ }^{s} \omega=\omega . \wedge s$, the process $Z\left(\tilde{\omega}, T^{s}\right)$ (denoted also by $Z(\tilde{\omega},$.$) ) defined by$

$$
Z\left(\tilde{\omega}, T^{s}\right): \Omega \times[s, T] \rightarrow \mathbb{R} ;\left(\omega^{\prime}, r\right) \mapsto Z_{r}\left(\tilde{\omega}, T^{s}\left(\omega^{\prime}\right)\right)
$$

belongs to $\mathbb{H}_{s}^{2}$. Moreover, if $Z \in \mathcal{A}$, then $Z\left(\tilde{\omega}, T^{s}\right) \in \mathcal{A}_{s}^{s}$.

This property also holds for all initial time $t \in[0, T]$. More precisely, let $s \in[t, T]$. Let $Z \in \mathbb{H}_{t}^{2}$ (resp., $\left.\mathcal{A}_{t}^{t}\right)$. For a.e. $\omega \in \Omega$, the process $Z\left({ }^{s} \omega,.\right)=\left(Z_{r}\left({ }^{s} \omega, T^{s}\right)\right)_{r \geq s}$ belongs to $\mathbb{H}_{s}^{2}$ (resp., $\left.\mathcal{A}_{s}^{s}\right)$.

Proof. Classically, we have $\mathbb{E}\left[\int_{s}^{T} Z_{r}^{2} d r\right]=\mathbb{E}\left[\mathbb{E}\left[\int_{s}^{T} Z_{r}^{2} d r \mid \mathcal{F}_{s}\right]\right]<+\infty$. Using the independence of $T^{s}$ with respect to $\mathcal{F}_{s}$ and the measurability of $S^{s}$ with respect to $\mathcal{F}_{s}$, we derive that

$$
\mathbb{E}\left[\int_{s}^{T} Z_{r}^{2} d r \mid \mathcal{F}_{s}\right]=\mathbb{E}\left[\int_{s}^{T} Z_{r}\left(S^{s}, T^{s}\right)^{2} d r \mid \mathcal{F}_{s}\right]=F\left(S^{s}\right)<+\infty, \quad P \text {-a.s. }
$$

where $F(\tilde{\omega}):=\mathbb{E}\left[\int_{s}^{T} Z_{r}\left(\tilde{\omega}, T^{s}(\cdot)\right)^{2} d r\right]$.

Let us now prove that the process $Z\left(\tilde{\omega}, T^{s}\right):\left(\omega^{\prime}, r\right) \mapsto Z_{r}\left(\tilde{\omega}, T^{s}\left(\omega^{\prime}\right)\right)$ is $\mathcal{P}^{s}-$ measurable. There exists a process indistinguishable of $\left(Z_{r}\right)$, still denoted by $\left(Z_{r}\right)$, which is measurable with respect to the predictable $\sigma$-algebra associated with the filtration generated by $W$ and $N$ (see [13, IV, section 79]). We can thus suppose in this proof (without loss of generality) that $\mathbb{F}$ (resp., $\mathbb{F}^{s}$ ) is the filtration generated by $W$ and $N$ (resp., $W^{s}$ and $N^{s}$ ), and $\mathcal{P}$ (resp., $\mathcal{P}^{s}$ ) is its associated predictable $\sigma$-algebra. Suppose we have shown that the map $\psi: \Omega \times[s, T] \rightarrow \Omega \times[0, T] ;\left(\omega^{\prime}, r\right) \mapsto$ $\left(\left(\tilde{\omega}, T^{s}\left(\omega^{\prime}\right)\right), r\right)$ is $\left(\mathcal{P}^{s}, \mathcal{P}\right)$-measurable. Now, we have $Z\left(\tilde{\omega}, T^{s}\right)\left(\omega^{\prime}, r\right)=Z \circ \psi\left(\omega^{\prime}, r\right)$ for each $\left(\omega^{\prime}, r\right) \in \Omega \times[s, T]$. Since $Z$ is $\mathcal{P}$-measurable, by composition, we derive that $Z\left(\tilde{\omega}, T^{s}\right)$ is $\mathcal{P}^{s}$-measurable.

It remains to show the $\left(\mathcal{P}^{s}, \mathcal{P}\right)$-measurability of $\psi$. Recall that the $\sigma$-algebra $\mathcal{P}$ is generated by the sets $H \times] v, T]$, where $v \in[0, T[$ and $H$ is of the form $H=$ 
$\left\{B_{t_{i}} \in A_{i}, \quad 1 \leq i \leq n\right\}$, where $A_{i} \in \mathcal{B}\left(\mathbb{R}^{2}\right)$ and $t_{1}<t_{2}<\cdots \leq v$. It is thus sufficient to show that $\left.\left.\psi^{-1}(H \times] v, T\right]\right) \in \mathcal{P}^{s}$. Note that $\left.\left.\left.\left.\psi^{-1}(H \times] v, T\right]\right)=H^{\prime} \times\right] v, T\right]$, where $H^{\prime}=\left\{\omega^{\prime} \in \Omega,\left(\tilde{\omega}, T^{s}\left(\omega^{\prime}\right)\right) \in H\right\}$. If there exists $i$ such that $t_{i} \leq s$ and $\tilde{\omega}_{t_{i}} \notin A_{i}$, then $H^{\prime}=\emptyset$. Otherwise, we have $H^{\prime}=\left\{\omega_{t_{i}}^{\prime}-\omega_{s}^{\prime} \in A_{i}\right.$ for all $i$ such that $\left.t_{i}>s\right\}$. Hence $H^{\prime} \in \mathcal{F}_{v}^{s}$, which implies that $\left.\left.\psi^{-1}(H \times] v, T\right]\right) \in \mathcal{P}^{s}$. The proof is thus complete.

Let $Z \in \mathbb{H}^{2}$. Let us give an intermediary time $s \in[0, T]$ and a fixed past path ${ }^{s} \omega$. Note that the Lebesgue integral $\left(\int_{s}^{u} Z_{r} d r\right)\left({ }^{s} \omega,.\right)$ is equal a.s. to the integral $\int_{s}^{u} Z_{r}\left({ }^{s} \omega,.\right) d r$. We now show that the stochastic integral $\left(\int_{s}^{u} Z_{r} d W_{r}\right)\left({ }^{s} \omega,.\right)$ coincides with the stochastic integral of the process $Z\left({ }^{s} \omega,.\right)$ with respect to the translated Brownian motion $W^{s}$, that is, $\int_{s}^{u} Z_{r}\left({ }^{s} \omega,.\right) d W_{r}^{s}$.

Lemma 5 (splitting properties for stochastic integrals). Let $s \in[0, T]$. Let $Z \in \mathbb{H}^{2}$ and $K \in \mathbb{H}_{\nu}^{2}$. There exists a $P$-null set $\mathcal{N}$ (which depends on s) such that for each $\omega \in \mathcal{N}^{c}$, and $\tilde{\omega}:={ }^{s} \omega$, we have $\left(Z_{r}\left(\tilde{\omega}, T^{s}\right)\right)_{r \geq s} \in \mathbb{H}_{s}^{2}$ and $\left(K_{r}\left(\tilde{\omega}, T^{s}\right)\right)_{r \geq s} \in \mathbb{H}_{s, \nu}^{2}$, and

$$
\begin{gathered}
\left(\int_{s}^{u} Z_{r} d W_{r}\right)\left(\tilde{\omega}, T^{s}\right)=\int_{s}^{u} Z_{r}\left(\tilde{\omega}, T^{s}\right) d W_{r}^{s}, \quad P \text {-a.s. } \\
\left(\int_{s}^{u} \int_{\mathbf{E}} K_{r}(e) \tilde{N}(d r, d e)\right)\left(\tilde{\omega}, T^{s}\right)=\int_{s}^{u} \int_{\mathbf{E}} K_{r}\left(\tilde{\omega}, T^{s}, e\right) \tilde{N}^{s}(d r, d e), \quad P \text {-a.s. }
\end{gathered}
$$

Remark 6. In the literature, the $s$-translated Brownian motion is often defined by $W_{v}^{\prime}:=W_{s+v}-W_{s}=W_{s+v}^{s}, 0 \leq v \leq T-s$. For each $Z \in \mathbb{H}_{s}^{2}$ and for each $u \geq s$, we have $\int_{s}^{u} Z_{r} d W_{r}^{s}=\int_{0}^{u-s} Z_{s+r} d W_{r}^{\prime}$ a.s. The use of $W^{s}$ thus allows us to avoid a change of time. The same remark holds for the Poisson random measure.

Note that equality (3.1) is equivalent to $\left(\int_{s}^{u} Z_{r} d W_{r}\right)\left(\tilde{\omega}, T^{s}\left(\omega^{\prime}\right)\right)=\left(\int_{s}^{u} Z_{r}\left(\tilde{\omega}, T^{s}\right)\right.$ $\left.d W_{r}^{s}\right)\left(\omega^{\prime}\right)$ for $P$-almost every $\omega^{\prime} \in \Omega$. The same remark holds for the second equality.

Proof. We shall only prove the first equality with the Brownian motion. The second one with the Poisson random measure can be shown by similar arguments.

Let us first show that equality (3.1) holds for a simple process. Let $a<T$ and let $H \in \mathbb{L}^{2}\left(\mathcal{F}_{a}\right)$. For each $\omega \equiv\left({ }^{s} \omega, \omega^{s}\right)=\left(S^{s}(\omega), T^{s}(\omega)\right) \in \Omega$, we have

$$
\begin{aligned}
\left(\int_{s}^{u} H \mathbf{1}_{] a, T]} d W_{r}\right)\left({ }^{s} \omega, \omega^{s}\right) & =H\left({ }^{s} \omega, \omega^{s}\right)\left(\omega_{u}^{s}-\omega_{a \wedge u}^{s}\right) \\
& =\left(\int_{s}^{u} H\left({ }^{s} \omega, T^{s}\right) \mathbf{1}_{] a, T]} d W_{r}^{s}\right)(\omega) .
\end{aligned}
$$

Let now $Z \in \mathbb{H}^{2}$. Let us show that $Z$ satisfies equality (3.1). The idea is to approximate $Z$ by an appropriate sequence of simple processes $\left(Z^{n}\right)_{n \in \mathbb{N}}$ so that the sequence $\left(Z^{n}\right)_{n \in \mathbb{N}}$ converges in $\mathbb{H}^{2}$ to $Z$ and that, for almost every past path ${ }^{s} \omega$, the sequence $\left(Z^{n}\left({ }^{s} \omega, T^{s}\right)\right)_{n \in \mathbb{N}}$ converges to $Z\left({ }^{s} \omega, T^{s}\right)$ in $\mathbb{H}_{s}^{2}$. For each $n \in \mathbb{N}^{*}$, define

$$
Z_{r}^{n}:=n \sum_{i=1}^{n-1}\left(\int_{\frac{(i-1) T}{n}}^{\frac{i T}{n}} Z_{u} d u\right) \mathbf{1}_{] \frac{i T}{n}, \frac{(i+1) T}{n}\right]}(r) .
$$

By inequality (A.2) in the appendix, we have $\int_{s}^{u}\left(Z_{r}^{n}(\omega)\right)^{2} d r \leq \int_{s}^{u} Z_{r}(\omega)^{2} d r$, and for each $\omega \in \Omega$ and $s \leq u, \int_{s}^{u}\left(Z_{r}^{n}(\omega)-Z_{r}(\omega)\right)^{2} d r \rightarrow 0$. Since $\int_{s}^{u} Z_{r}^{2} d r \in L^{1}(\Omega)$, it follows, by the Lebesgue theorem for the conditional expectation, that

$$
\mathbb{E}\left[\int_{s}^{u}\left(Z_{r}^{n}-Z_{r}\right)^{2} d r \mid \mathcal{F}_{s}\right] \rightarrow 0
$$


except on a $P$-null set $\mathcal{N}$. Since $S^{s}$ is $\mathcal{F}_{s}$-measurable and $T^{s}$ is independant of $\mathcal{F}_{s}$, there exists a $P$-null set included in the previous one, such that for each $\omega \in \mathcal{N}^{c}$, setting $\tilde{\omega}={ }^{s} \omega$, we have

$$
\begin{aligned}
\mathbb{E}\left[\int_{s}^{u}\left(Z_{r}^{n}-Z_{r}\right)^{2} d r \mid \mathcal{F}_{s}\right](\tilde{\omega}) & =\mathbb{E}\left[\int_{s}^{u}\left(Z_{r}^{n}\left(\tilde{\omega}, T^{s}\right)-Z_{r}\left(\tilde{\omega}, T^{s}\right)\right)^{2} d r\right] \\
& =\mathbb{E}\left[\left(\int_{s}^{u} Z_{r}^{n}\left(\tilde{\omega}, T^{s}\right) d W_{r}^{s}-\int_{s}^{u} Z_{r}\left(\tilde{\omega}, T^{s}\right) d W_{r}^{s}\right)^{2}\right] .
\end{aligned}
$$

The second equality follows by the classical isometry property. Now, for each square integrable martingale $M, M^{2}-\langle M\rangle$ is a martingale. Hence, for each $\omega \in \mathcal{N}^{c}$, where $\mathcal{N}$ is a $P$-null set included in the previous one, setting $\tilde{\omega}={ }^{s} \omega$, we have

$$
\begin{aligned}
\mathbb{E} & {\left[\int_{s}^{u}\left(Z_{r}^{n}-Z_{r}\right)^{2} d r \mid \mathcal{F}_{s}\right](\tilde{\omega}) } \\
= & \mathbb{E}\left[\left(\int_{s}^{u} Z_{r}^{n} d W_{r}-\int_{s}^{u} Z_{r} d W_{r}\right)^{2} \mid \mathcal{F}_{s}\right](\tilde{\omega}) \\
= & \mathbb{E}\left[\left(\left(\int_{s}^{u} Z_{r}^{n} d W_{r}\right)\left(\tilde{\omega}, T^{s}\right)-\left(\int_{s}^{u} Z_{r} d W_{r}\right)\left(\tilde{\omega}, T^{s}\right)\right)^{2}\right] .
\end{aligned}
$$

For each $n \in \mathbb{N}^{*}$, since $Z^{n}$ is a simple process, it satisfies equality (3.1) everywhere, that is, $\left(\int_{s}^{u} Z_{r}^{n} d W_{r}\right)\left(\tilde{\omega}, T^{s}\right)=\int_{s}^{u} Z_{r}^{n}\left(\tilde{\omega}, T^{s}\right) d W_{r}^{s}$. By the convergence property (3.2), equalities (3.3) and (3.4), and the uniqueness property of the limit in $L^{2}$, we derive that for each $\omega \in \mathcal{N}^{c}$, setting $\tilde{\omega}={ }^{s} \omega$, equality (3.1) holds. The proof is thus complete.

Using the above lemmas, we now show that for each $s \geq t$, for almost every $\omega \in \Omega$, setting $\tilde{\omega}={ }^{s} \omega$, the process $Y^{\alpha, t, x}\left(\tilde{\omega}, T^{s}\right)$ coincides with the solution of the reflected BSDE on $\Omega \times[s, T]$, associated with driver $f^{\alpha\left(\tilde{\omega}, T^{s}\right), s, \eta(\tilde{\omega})}$ with obstacle $\bar{h}\left(r, X_{r}^{\alpha\left(\tilde{\omega}, T^{s}\right), s, X_{s}^{\alpha(\tilde{\omega}), t, x}(\tilde{\omega})}\right)$ and filtration $\mathbb{F}^{s}$, and driven by $W^{s}$ and $\tilde{N}^{s}$.

To simplify notation, $T^{s}$ will be replaced by $\cdot$ in the following. In particular $Y^{\alpha, t, x}\left(\tilde{\omega}, T^{s}\right)$ will be simply denoted by $Y^{\alpha, t, x}(\tilde{\omega},$.$) .$

Theorem 7 (splitting properties for the forward-backward system). Let $t \in$ $[0, T], \alpha \in \mathcal{A}_{t}^{t}$, and $s \in[t, T]$. There exists a P-null set $\mathcal{N}$ (which depends on $t$ and s) such that for each $\omega \in \mathcal{N}^{c}$, setting $\tilde{\omega}={ }^{s} \omega$, the following properties hold:

- There exists an unique solution $\left(X_{r}^{\alpha(\tilde{\omega}, \cdot), s, \eta(\tilde{\omega})}\right)_{s \leq r \leq T}$ in $\mathcal{S}_{s}^{2}$ of the following $S D E$ :

$$
\begin{aligned}
X_{r}^{\alpha(\tilde{\omega}, \cdot), s, \eta(\tilde{\omega})}= & \eta(\tilde{\omega})+\int_{s}^{r} b\left(X_{v}^{\alpha(\tilde{\omega}, \cdot), s, \eta(\tilde{\omega})}, \alpha_{v}(\tilde{\omega}, \cdot)\right) d v \\
& +\int_{s}^{r} \sigma\left(X_{v}^{\alpha(\tilde{\omega}, \cdot), s, \eta(\tilde{\omega})}, \alpha_{v}(\tilde{\omega}, \cdot)\right) d W_{v}^{s} \\
& +\int_{s}^{r} \int_{\mathbf{E}} \beta\left(X_{v^{-}}^{\alpha(\tilde{\omega}, \cdot), s, \eta(\tilde{\omega})}, \alpha_{v}(\tilde{\omega}, \cdot), e\right) \tilde{N}^{s}(d v, d e),
\end{aligned}
$$

where $\eta(\tilde{\omega}):=X_{s}^{\alpha(\tilde{\omega}), t, x}(\tilde{\omega})$. We also have $X_{r}^{\alpha, t, x}(\tilde{\omega},)=.X_{r}^{\alpha(\tilde{\omega}, \cdot), s, \eta(\tilde{\omega})}, s \leq$ $r \leq T$ P-a.s.

- There exists an unique solution $\left(Y_{r}^{\alpha(\tilde{\omega}, \cdot), s, \eta(\tilde{\omega})}, Z_{r}^{\alpha(\tilde{\omega}, \cdot), s, \eta(\tilde{\omega})}, K_{r}^{\alpha(\tilde{\omega}, \cdot), s, \eta(\tilde{\omega})}\right)_{s \leq r \leq T}$ in $\mathcal{S}_{s}^{2} \times \mathbb{H}_{s}^{2} \times \mathbb{H}_{s, \nu}^{2}$ of the reflected BSDE on $\Omega \times[s, T]$ driven by $W^{s}$ and $\tilde{N}^{s}$ 
and associated with filtration $\mathbb{F}^{s}$, driver $f^{\alpha(\tilde{\omega}, .), s, \eta(\tilde{\omega})}$, and obstacle $\bar{h}\left(r, X_{r}^{\alpha(\tilde{\omega}, \cdot), s, \eta(\tilde{\omega})}\right)$. We have the following:

$$
\begin{aligned}
Y_{r}^{\alpha, t, x}(\tilde{\omega}, .) & =Y_{r}^{\alpha(\tilde{\omega}, .), s, \eta(\tilde{\omega})}, \quad s \leq r \leq T, \quad P \text {-a.s. } \\
Z_{r}^{\alpha, t, x}(\tilde{\omega}, .) & =Z_{r}^{\alpha(\tilde{\omega}, \cdot), s, \eta(\tilde{\omega})} \text { and } K_{r}^{\alpha, t, x}(\tilde{\omega}, .) \\
& =K_{r}^{\alpha(\tilde{\omega}, \cdot), s, \eta(\tilde{\omega})}, \quad s \leq r \leq T, \quad d P \otimes d r \text {-a.s. } \\
Y_{s}^{\alpha, t, x}(\tilde{\omega}, .) & =Y_{s}^{\alpha(\tilde{\omega}, \cdot), s, \eta(\tilde{\omega})}=u^{\alpha(\tilde{\omega}, \cdot)}(s, \eta(\tilde{\omega})), \quad P \text {-a.s. }
\end{aligned}
$$

Proof. Recall that by Lemma 4, there exists a $P$-null set $\mathcal{N}$ such that for each $\omega \in \mathcal{N}^{c}$, the process $\alpha\left({ }^{s} \omega, \cdot\right):=\left(\alpha_{r}\left({ }^{s} \omega, T_{s}\right)\right)_{r \geq s}$ belongs to $\mathcal{A}_{s}^{s}$.

Let us show the first assertion. To simplify the exposition, we suppose that there is no Poisson random measure. There exists a $P$-null set, still denoted by $\mathcal{N}$, included in the above one such that for each $\omega \in \mathcal{N}^{c}$, setting $\tilde{\omega}={ }^{s} \omega$,

$$
X_{r}^{\alpha, t, x}(\tilde{\omega}, .)=\eta(\tilde{\omega})+\int_{s}^{r} b\left(X_{v}^{\alpha, t, x}(\tilde{\omega}, .), \alpha_{v}(\tilde{\omega}, .)\right) d v+\left(\int_{s}^{r} \sigma\left(X_{v}^{\alpha, t, x}, \alpha_{v}\right) d W_{v}\right)(\tilde{\omega}, .),
$$

on $[s, T], P$-a.s. Now, by the first equality in Lemma 5 , there exists a $P$-null set $\mathcal{N}$ such that for each $\omega \in \mathcal{N}^{c}$, setting $\tilde{\omega}={ }^{s} \omega$, we have

$$
\left(\int_{s}^{r} \sigma\left(X_{v}^{\alpha, t, x}, \alpha_{v}\right) d W_{v}\right)(\tilde{\omega}, .)=\int_{s}^{r} \sigma\left(X_{v}^{\alpha(\tilde{\omega}, \cdot), s, \eta(\tilde{\omega})}, \alpha_{v}(\tilde{\omega}, \cdot)\right) d W_{v}^{s}, \quad P \text {-a.s. }
$$

which implies that the process $\left(X_{r}^{\alpha, t, x}(\tilde{\omega}, \cdot)\right)_{r \in[s, T]}$ is a solution of SDE (3.5), and then, by uniqueness of the solution of this SDE, we have $X_{r}^{\alpha, t, x}(\tilde{\omega},)=.X_{r}^{\alpha(\tilde{\omega}, .), s, \eta(\tilde{\omega})}$, $s \leq r \leq T, P$-a.s.

Let us show the second assertion. First, note that since the filtration $\mathbb{F}^{s}$ is the completed filtration of the natural filtration of $W^{s}$ and $\tilde{N}^{s}$ (with respect to the initial $\sigma$-algebra $\mathcal{B}(\Omega)$ ), we have a martingale representation theorem for $\mathbb{F}^{s}$ martingales with respect to $W^{s}$ and $\tilde{N}^{s}$. Hence, there exists an unique solution $\left(Y_{r}^{\alpha(\tilde{\omega}, \cdot), s, \eta(\tilde{\omega})}, Z_{r}^{\alpha(\tilde{\omega}, \cdot), s, \eta(\tilde{\omega})}, K_{r}^{\alpha(\tilde{\omega}, \cdot), s, \eta(\tilde{\omega})}\right)_{s \leq r \leq T}$ in $\mathcal{S}_{s}^{2} \times \mathbb{H}_{s}^{2} \times \mathbb{H}_{s, \nu}^{2}$ of the reflected BSDE on $\Omega \times[s, T]$ driven by $W^{s}$ and $\tilde{N}^{s}$ and associated with filtration $\mathbb{F}^{s}$ and with obstacle $\bar{h}\left(r, X_{r}^{\alpha(\tilde{\omega}, \cdot), s, \eta(\tilde{\omega})}\right)$. Equalities (3.6) then follow from similar arguments as above together with the uniqueness of the solution of a Lipschitz RBSDE. Equality (3.7) is obtained by taking $r=s$ in equality (3.6) and by using the definition of $u^{\alpha(\tilde{\omega}, .)}$.

Remark 8. In the above proofs, we have treated the $P$-null sets issues carefully. We stress that all the filtrations are completed with respect to $\mathcal{B}(\Omega)$ and $P$. The underlying probability space is thus always the completion of the initial probability space $(\Omega, \mathcal{B}(\Omega), P)$ (that is, $\left.\left(\Omega, \mathcal{F}_{T}, P\right)\right)$. Note that the $P$-null sets remain always the same, which is particularly important for stochastic integrals (see Lemma 5) and also for BSDEs because the solution of a BSDE is unique up to a P-null set.

Moreover, in the proof of Lemma 5, the choice of the sequence of step functions approximating the process $Z$ is appropriate to handle the issues of $P$-null sets.

Note that Theorem 7 applied to the simpler case when $\alpha \in \mathcal{A}_{s}^{s}$ ensures that the solution of (2.8) (with $t$ replaced by $s$ ) coincides on $[s, T] \times \Omega$ with the solution in $\mathcal{S}^{2} \times \mathbb{H}^{2} \times \mathbb{H}_{\nu}^{2}$ of the reflected BSDE similar to (2.8) but driven by $W$ and $\tilde{N}$ instead of $W^{s}$ and $\tilde{N}^{s}$, and associated with $\mathbb{F}$. 
3.2. Measurability properties and $\varepsilon$-optimal controls. We need to show a measurability property of the function $u^{\alpha}(t, x)$ with respect to control $\alpha$ and initial condition $x$. To this purpose, we first provide a preliminary result, which will allow us to handle the nonlinearity of the expectation.

Proposition 9. Let $(\Omega, \mathcal{F}, P)$ be a probability space. For each $q \geq 0$, we denote by $L^{q}$ the set $L^{q}(\Omega, \mathcal{F}, P)$. Suppose that the Hilbert space $L^{2}$ equipped with the usual scalar product is separable.

Let $g: \mathbb{R} \rightarrow \mathbb{R}$ be a Borelian function such that $|g(x)| \leq C\left(1+|x|^{p}\right)$ for each real $x$ with $p \geq 0$. The map $\varphi^{g}$ defined by

$$
\varphi^{g}: L^{2 p} \cap L^{2} \rightarrow L^{2} ; \xi \mapsto g \circ \xi(=g(\xi))
$$

is then $\mathcal{B}^{\prime}\left(L^{2 p} \cap L^{2}\right) / \mathcal{B}\left(L^{2}\right)$-measurable, where $\mathcal{B}\left(L^{2}\right)$ is the Borelian $\sigma$-algebra on $L^{2}$, and $\mathcal{B}^{\prime}\left(L^{2 p} \cap L^{2}\right)$ is the $\sigma$-algebra induced by $\mathcal{B}\left(L^{2}\right)$ on $L^{2 p} \cap L^{2}$.

The proof of this proposition is postponed until the appendix.

Using this result, we now show the following measurability property.

Theorem 10. Let $s \in[0, T]$. The map $(\alpha, x) \mapsto u^{\alpha}(s, x) ; \mathcal{A}_{s}^{s} \times \mathbb{R} \rightarrow \mathbb{R}$, is $\mathcal{B}^{\prime}\left(\mathcal{A}_{s}^{s}\right) \otimes \mathcal{B}(\mathbb{R}) / \mathcal{B}(\mathbb{R})$-measurable, where $\mathcal{B}^{\prime}\left(\mathcal{A}_{s}^{s}\right)$ denotes the $\sigma$-algebra induced by $\mathcal{B}\left(\mathbb{H}_{s}^{2}\right)$ on $\mathcal{A}_{s}^{s}$.

Proof. Recall that $u^{\alpha}(s, x)=Y_{s, T}^{\alpha, s, x}\left[g\left(X_{T}^{\alpha, s, x}\right)\right]$ is also denoted by $Y_{s, T}^{\alpha, s, x}\left[\bar{h}\left(., X^{\alpha, s, x}\right)\right]$. Let $x^{1}, x^{2} \in \mathbb{R}$, and $\alpha^{1}, \alpha^{2} \in \mathcal{A}_{s}^{s}$. By classical estimates on diffusion processes and the assumptions made on the coefficients, we get

$$
\mathbb{E}\left[\sup _{r \geq s}\left|X_{r}^{\alpha^{1}, s, x^{1}}-X_{r}^{\alpha^{2}, s, x^{2}}\right|^{2}\right] \leq C\left(\left\|\alpha^{1}-\alpha^{2}\right\|_{\mathbb{H}_{s}^{2}}^{2}+\left|x^{1}-x^{2}\right|^{2}\right) .
$$

We introduce the map $\Phi: \mathcal{A}_{s}^{s} \times \mathbb{R} \times \mathcal{S}_{s}^{2} \times L_{s}^{2} \rightarrow \mathcal{S}_{s}^{2} ;(\alpha, x, \zeta ., \xi) \mapsto Y_{s, T}^{\alpha, s, x}[\eta$., $\xi]$, where $Y_{s, T}^{\alpha, s, x}[\zeta ., \xi]$ denotes here the solution at time $s$ of the reflected BSDE associated with driver $f^{\alpha, s, x}:=\left(f\left(\alpha_{r}, r, X_{r}^{\alpha, s, x},.\right) \mathbf{1}_{r \geq s}\right)$, obstacle $\left(\eta_{s}\right)_{s<T}$, and terminal condition $\xi$.

By the estimates on RBSDEs (see the appendix in [14]), using the Lipschitz property of $f$ w.r.t. $x, \alpha$ and estimates (3.8), for all $x^{1}, x^{2} \in \mathbb{R}, \alpha^{1}, \alpha^{2} \in \mathcal{A}_{s}^{s}, \eta^{1}, \eta^{2} \in S_{s}^{2}$ and $\xi^{1}, \xi^{2} \in L_{s}^{2}$, we have

$$
\begin{aligned}
\mid Y_{s, T}^{\alpha^{1}, s, x^{1}}\left[\eta^{1}, \xi^{1}\right]- & \left.Y_{s, T}^{\alpha^{2}, s, x^{2}}\left[\eta^{2}, \xi^{2}\right]\right|^{2} \\
& \leq C\left(\left\|\alpha^{1}-\alpha^{2}\right\|_{\mathbb{H}_{s}^{2}}^{2}+\left|x^{1}-x^{2}\right|^{2}+\left\|\eta{ }^{1}-\eta_{\cdot}^{2}\right\|_{S_{s}^{2}}^{2}+\left\|\xi^{1}-\xi^{2}\right\|_{L_{s}^{2}}^{2}\right) .
\end{aligned}
$$

The map $\Phi$ is thus Lipschitz-continuous with respect to the norm $\|\cdot\|_{\mathbb{H}_{s}^{2}}^{2}+|\cdot|^{2}+$ $\|\cdot\|_{\mathcal{S}_{s}^{2}}^{2}+\|\cdot\|_{L_{s}^{2}}^{2}$.

Recall that by assumption, $|h(t, x)| \leq C\left(1+|x|^{p}\right)$, and that $h$ is Lipschitz continuous with respect to $x$ uniformly in $t$. One can derive that the map $S_{s}^{2 p} \cap S_{s}^{2} \rightarrow S_{s}^{2}$, $\eta$. $\mapsto h\left(., \eta\right.$.) is Lipschitz-continuous for the norm $\|.\|_{S_{s}^{2}}^{2}$ and thus Borelian, $S_{s}^{2}$ being equipped with the Borelian $\sigma$-algebra $\mathcal{B}\left(S_{s}^{2}\right)$ and its subspace $S_{s}^{2 p} \cap S_{s}^{2}$ with the $\sigma$-algebra induced by $\mathcal{B}\left(S_{s}^{2}\right)$.

Moreover, by Lemma 25, the Hilbert space $L_{s}^{2}$ is separable. We can thus apply Proposition 9 and get that the map $L_{s}^{2 p} \cap L_{s}^{2} \rightarrow L_{s}^{2}, \xi \mapsto g(\xi)$ is Borelian.

We thus derive that the map $(\alpha, x) \mapsto\left(\alpha, x, h\left(., X^{\alpha, s, x}\right), g\left(X_{T}^{\alpha, s, x}\right)\right)$ defined on $\mathcal{A}_{s}^{s} \times \mathbb{R}$ and valued in $\mathcal{A}_{s}^{s} \times \mathbb{R} \times \mathcal{S}_{s}^{2} \times L_{s}^{2}$ is $\mathcal{B}^{\prime}\left(\mathcal{A}_{s}^{s}\right) \otimes \mathcal{B}(\mathbb{R}) / \mathcal{B}^{\prime}\left(\mathcal{A}_{s}^{s}\right) \otimes \mathcal{B}(\mathbb{R}) \otimes \mathcal{B}\left(\mathcal{S}_{s}^{2}\right) \otimes \mathcal{B}\left(L_{s}^{2}\right)$ measurable. By composition, it follows that the map $(\alpha, x) \mapsto Y_{s, T}^{\alpha, s, x}\left[h\left(., X_{.}^{\alpha, s, x}\right)\right.$, $\left.g\left(X_{T}^{\alpha, s, x}\right)\right]=u^{\alpha}(s, x)$ is measurable. 
For each $(t, s)$ with $s \geq t$, we introduce the set $\mathcal{A}_{s}^{t}$ of restrictions to $[s, T]$ of the controls in $\mathcal{A}_{t}^{t}$. They can also be identified to the controls $\alpha$ in $\mathcal{A}_{t}^{t}$ which are equal to 0 on $[t, s]$.

Let $\eta \in L^{2}\left(\mathcal{F}_{s}^{t}\right)$. Since $\eta$ is $\mathcal{F}_{s}$-measurable, up to a $P$-null set, it can be written as a measurable map, still denoted by $\eta$, of the past trajectory ${ }^{s} \omega$. (See the argument used in the proof of Lemma 25 for details.) For each $\omega \in \Omega$, by using the definition of the function $u$, we have

$$
u\left(s, \eta\left({ }^{s} \omega\right)\right)=\sup _{\alpha \in \mathcal{A}_{s}^{s}} u^{\alpha}\left(s, \eta\left({ }^{s} \omega\right)\right) .
$$

By Theorem 10 together with a measurable selection theorem, we show the existence of nearly optimal controls for (3.9) satisfying some specific measurability properties.

Theorem 11 (existence of $\varepsilon$-optimal controls). Let $t \in[0, T], s \in[t, T[$, and $\eta \in L^{2}\left(\mathcal{F}_{s}^{t}\right)$. Let $\varepsilon>0$. There exists $\alpha^{\varepsilon} \in \mathcal{A}_{s}^{t}$ such that, for almost every $\omega \in \Omega$, $\alpha^{\varepsilon}\left({ }^{s} \omega, T^{s}\right)$ is $\varepsilon$-optimal for Problem (3.9), in the sense that

$$
u\left(s, \eta\left({ }^{s} \omega\right)\right) \leq u^{\alpha^{\varepsilon}\left({ }^{s} \omega, T^{s}\right)}\left(s, \eta\left({ }^{s} \omega\right)\right)+\varepsilon .
$$

Proof. Without loss of generality, we may assume that $t=0$. We introduce the space ${ }^{s} \Omega:=\left\{\left(\omega_{r}\right)_{0 \leq r \leq s} ; \omega \in \Omega\right\}$, equipped with its Borelian $\sigma$-algebra denoted by $\mathcal{B}\left({ }^{s} \Omega\right)$, and the probability measure ${ }^{s} P$, which corresponds to the image of $P$ by ${ }^{s} S: \Omega \rightarrow{ }^{s} \Omega ; \omega \mapsto\left(\omega_{r}\right)_{r \leq s}$. The Hilbert space $\mathbb{H}_{s}^{2}$ of square-integrable predictable processes on $\Omega^{s} \times[s, T]$, equipped with the norm $\|\cdot\|_{\mathbb{H}_{s}^{2}}$, is separable (see Lemma 25). Moreover, $\mathcal{A}_{s}^{s}$ is a closed subset of $\mathbb{H}_{s}^{2}$. Also, the space ${ }^{s} \Omega$ of paths (RCLL) before $s$ is Polish for the Skorohod metric. Now, as seen above, since $\eta$ is $\mathcal{F}_{s^{-}}$-measurable, up to a $P$-null set, we can suppose that it is of the form $\eta \circ S^{s}$, where $\eta$ is $\mathcal{B}\left({ }^{s} \Omega\right)$ measurable. Moreover, by Theorem 10, the map $(\tilde{\omega}, \alpha) \mapsto u^{\alpha}(s, \eta(\tilde{\omega}))$ is $\mathcal{B}\left({ }^{s} \Omega\right) \otimes$ $\mathcal{B}\left(\mathcal{A}_{s}^{s}\right)$-measurable with respect to $(x, \alpha)$. We can thus apply [6, Proposition 7.50] to the problem (3.9). Hence, there exists a map $\underline{\alpha}^{\varepsilon}:{ }^{s} \Omega \mapsto \mathcal{A}_{s}^{s} ; \tilde{\omega} \mapsto \underline{\alpha}^{\varepsilon}(\tilde{\omega}, \cdot)$, which is universally measurable, that is $\mathcal{U}\left({ }^{s} \Omega\right) / \mathcal{B}\left(\mathcal{A}_{s}^{s}\right)$-measurable, and such that

$$
u(s, \eta(\tilde{\omega})) \leq u^{\underline{\alpha}^{\varepsilon}}(\tilde{\omega}, \cdot)(s, \eta(\tilde{\omega}))+\varepsilon \quad \forall \tilde{\omega} \in{ }^{s} \Omega .
$$

Here, $\mathcal{U}\left({ }^{s} \Omega\right)$ denotes the universal $\sigma$-algebra on ${ }^{s} \Omega$. Let us now apply Lemma 26 to $X={ }^{s} \Omega$, to $E=\mathbb{H}_{s}^{2}$, and to probability $Q=P^{s}$. By definition of $\mathcal{U}\left({ }^{s} \Omega\right.$ ) (see, e.g., [6]), we have $\mathcal{U}\left({ }^{s} \Omega\right) \subset \mathcal{B}_{Q}\left({ }^{s} \Omega\right)$, where $\mathcal{B}_{Q}\left({ }^{s} \Omega\right)$ denotes the completion of $\mathcal{B}\left({ }^{s} \Omega\right)$ with respect to $Q$. Hence, there exists a map $\hat{\alpha}^{\varepsilon}:{ }^{s} \Omega \mapsto \mathcal{A}_{s}^{s} ; \tilde{\omega} \mapsto \hat{\alpha}^{\varepsilon}(\tilde{\omega}, \cdot)$ which is Borelian, that is $\mathcal{B}\left({ }^{s} \Omega\right) / \mathcal{B}\left(\mathcal{A}_{s}^{s}\right)$-measurable, and such that

$$
\hat{\alpha}^{\varepsilon}(\tilde{\omega}, \cdot)=\underline{\alpha}^{\varepsilon}(\tilde{\omega}, \cdot) \quad \text { for } \quad{ }^{s} P \text {-almost every } \tilde{\omega} \in{ }^{s} \Omega .
$$

Since $\mathbb{H}_{s}^{2}$ is a separable Hilbert space, for each $\tilde{\omega}$, we have $\hat{\alpha}_{u}^{\varepsilon}(\tilde{\omega}, \omega)=\sum_{i} \beta^{i, \varepsilon}(\tilde{\omega}) e_{u}^{i}(\omega)$ $d P(\omega) \otimes d u$-a.s., where $\beta^{i, \varepsilon}(\tilde{\omega})=<\hat{\alpha}^{\varepsilon}(\tilde{\omega}, \cdot), e^{i}(\cdot)>_{\mathbb{H}_{s}^{2}}$ and $\left\{e^{i}, i \in \mathbb{N}\right\}$ is a countable orthonormal basis of $\mathbb{H}_{s}^{2}$. Note that $\beta^{i, \varepsilon}$ is Borelian, that is, $\mathcal{B}\left({ }^{s} \Omega\right) / \mathcal{B}(\mathbb{R})$-measurable. Let $\bar{\alpha}^{\varepsilon}:{ }^{s} \Omega \mapsto \mathcal{A}_{s}^{s} ; \tilde{\omega} \mapsto \bar{\alpha}^{\varepsilon}(\tilde{\omega}, \cdot)=\sum_{i} \beta^{i, \varepsilon}(\tilde{\omega}) e^{i}(\cdot)$. It is Borelian, that is, $\mathcal{B}\left({ }^{s} \Omega\right) / \mathcal{B}\left(\mathcal{A}_{s}^{s}\right)-$ measurable.

We now define a process $\alpha^{\varepsilon}$ on $[0, T] \times \Omega$ by $\alpha_{r}^{\varepsilon}(\omega):=\sum_{i} \beta^{i, \varepsilon}\left(S^{s}(\omega)\right) e^{i}(\omega)$. It remains to prove that it is $\mathcal{P}$-measurable. Note that $\beta^{i, \varepsilon} \circ S^{s}$ is $\mathcal{F}_{s}$-measurable by composition. Since the process $\left(e_{u}^{i}\right)_{s \leq u \leq T}$ is $\mathcal{P}^{s}$-measurable, the process $\left(\beta^{i, \varepsilon} \circ S^{s}\right) e_{u}^{i}$ 
is $\mathcal{P}$-measurable. Indeed, if we take $e^{i}$ of the form $e_{u}^{i}=H \mathbf{1}_{] r, T]}(u)$ with $r \geq s$ and $H$ a random variable $\mathcal{F}_{r}^{s}$-measurable, then the random variable $\left(\beta^{i, \varepsilon} \circ S^{s}\right) H$ is $\mathcal{F}_{r^{-}}$ measurable and hence the process $\left(\beta^{i, \varepsilon} \circ S^{s}\right) H \mathbf{1}_{] r, T]}$ is $\mathcal{P}$-measurable. The process $\alpha^{\varepsilon}$ is thus $\mathcal{P}$-measurable.

Note also that $\alpha^{\varepsilon}\left(\tilde{\omega}, T^{s}(\omega)\right)=\sum_{i} \beta^{i, \varepsilon}(\tilde{\omega}) e^{i}(\tilde{\omega}, \omega)$. Now, we have $e^{i}\left(\tilde{\omega}, T^{s}(\omega)\right)=$ $e^{i}(\omega)$ because $e^{i}(\omega)$ depends on $\omega$ only through $T^{s}(\omega)$. Hence, $\alpha^{\varepsilon}\left(\tilde{\omega}, T^{s}(\omega)\right)=$ $\bar{\alpha}^{\varepsilon}(\tilde{\omega}, \omega)$, which completes the proof.

3.3. A Fatou lemma for reflected BSDEs. We establish a Fatou lemma for reflected BSDEs, where the limit involves both terminal condition and terminal time. This result will be used to prove a super- (resp., sub-) optimality principle involving the l.s.c. (resp., u.s.c.) envelope of the value function $u$ (see Theorem 17). We first introduce some notation.

A function $f$ is said to be a Lipschitz driver if $f:[0, T] \times \Omega \times \mathbb{R}^{2} \times L_{\nu}^{2} \rightarrow$ $\mathbb{R}(\omega, t, y, z, k(\cdot)) \mapsto f(\omega, t, y, z, k(\cdot))$ is $\mathcal{P} \otimes \mathcal{B}\left(\mathbb{R}^{2}\right) \otimes \mathcal{B}\left(L_{\nu}^{2}\right)$-measurable, uniformly Lipschitz with respect to $y, z, k(\cdot)$, and such that $f(., 0,0,0) \in \mathbb{H}^{2}$.

A Lipschitz driver $f$ is said to satisfy Assumption 12 if the following holds.

Assumption 12. Assume that $d P \otimes d t$-a.s for each $\left(y, z, k_{1}, k_{2}\right) \in \mathbb{R}^{2} \times\left(L_{\nu}^{2}\right)^{2}$,

$$
f\left(t, y, z, k_{1}\right)-f\left(t, y, z, k_{2}\right) \geq\left\langle\gamma_{t}^{y, z, k_{1}, k_{2}}, k_{1}-k_{2}\right\rangle_{\nu}
$$

with $\gamma:[0, T] \times \Omega \times \mathbb{R}^{2} \times\left(L_{\nu}^{2}\right)^{2} \rightarrow L_{\nu}^{2} ;\left(\omega, t, y, z, k_{1}, k_{2}\right) \mapsto \gamma_{t}^{y, z, k_{1}, k_{2}}(\omega,$.$) , supposed to$ be $\mathcal{P} \otimes \mathcal{B}\left(\mathbb{R}^{2}\right) \otimes \mathcal{B}\left(\left(L_{\nu}^{2}\right)^{2}\right)$-measurable, uniformly bounded in $L_{\nu}^{2}$, and satisfying $d P(\omega) \otimes$ $d t \otimes d \nu(e)$-a.s., for each $\left(y, z, k_{1}, k_{2}\right) \in \mathbb{R}^{2} \times\left(L_{\nu}^{2}\right)^{2}$, the inequality $\gamma_{t}^{y, z, k_{1}, k_{2}}(\omega, e) \geq-1$.

This assumption ensures the comparison theorem for BSDEs with jumps (see [21, Theorem 4.2]).

Let $\left(\eta_{t}\right)$ be a given RCLL obstacle process in $\mathcal{S}^{2}$ and let $f$ be a given Lipschitz driver. In the following, we will consider the case when the terminal time is a stopping time $\theta \in \mathcal{T}$ and the terminal condition is a random variable $\xi$ in $L^{2}\left(\mathcal{F}_{\theta}\right)$. In this case, the solution, denoted $\left(Y_{., \theta}(\xi), Z_{., \theta}(\xi), k_{., \theta}(\xi)\right)$, of the reflected BSDEs associated with terminal stopping time $\theta$, driver $f$, obstacle $\left(\eta_{s}\right)_{s<\theta}$, and terminal condition $\xi$ is defined as the unique solution in $\mathcal{S}^{2} \times \mathbb{H}^{2} \times \mathbb{H}_{\nu}^{2}$ of the reflected BSDE with terminal time $T$, driver $f(t, y, z, k) \mathbf{1}_{\{t \leq \theta\}}$, terminal condition $\xi$, and obstacle $\eta_{t} \mathbf{1}_{t<\theta}+\xi \mathbf{1}_{t \geq \theta}$. Note that $Y_{t, \theta}(\xi)=\xi, Z_{t, \theta}(\xi)=0, k_{t, \theta}(\xi)=0$ for $t \geq \theta$.

We first prove a continuity property for reflected BSDEs where the limit involves both terminal condition and terminal time.

Proposition 13 (a continuity property for reflected BSDEs). Let $T>0$. Let $\left(\eta_{t}\right)$ be an RCLL process in $\mathcal{S}^{2}$. Let $f$ be a given Lipschitz driver. Let $\left(\theta^{n}\right)_{n \in \mathbb{N}}$ be a non increasing sequence of stopping times in $\mathcal{T}$, converging a.s. to $\theta \in \mathcal{T}$ as $n$ tends to $\infty$. Let $\left(\xi^{n}\right)_{n \in \mathbb{N}}$ be a sequence of random variables such that $\mathbb{E}\left[\sup _{n}\left(\xi^{n}\right)^{2}\right]<+\infty$, and for each $n, \xi^{n}$ is $\mathcal{F}_{\theta^{n}}$-measurable. Suppose that $\xi^{n}$ converges a.s. to an $\mathcal{F}_{\theta}$-measurable random variable $\xi$ as $n$ tends to $\infty$. Suppose that

$$
\eta_{\theta} \leq \xi \quad \text { a.s. }
$$

Let $Y_{., \theta^{n}}\left(\xi^{n}\right) ; Y_{., \theta}(\xi)$ be the solutions of the reflected BSDEs associated with driver $f$, obstacle $\left(\eta_{s}\right)_{s<\theta^{n}}\left(\right.$ resp.. $\left.\left(\eta_{s}\right)_{s<\theta}\right)$, terminal time $\theta^{n}$ (resp., $\left.\theta\right)$, and terminal condition $\xi^{n}$ (resp., $\xi$ ). We have

$$
Y_{0, \theta}(\xi)=\lim _{n \rightarrow+\infty} Y_{0, \theta^{n}}\left(\xi^{n}\right) \text { a.s. }
$$

When for each $n, \theta_{n}=\theta$ a.s., the result still holds without assumption (3.10). 
By similar arguments as in the Brownian case (see, e.g., [16]), one can prove the following estimate on reflected BSDEs, which will be used in the proof of the above proposition.

Lemma 14. Let $\xi^{1}, \xi^{2} \in L^{2}\left(\mathcal{F}_{T}\right)$ and $\left(\eta_{t}^{1}\right),\left(\eta_{t}^{2}\right) \in \mathcal{S}^{2}$. Let $f^{1}, f^{2}$ be Lipschitz drivers with Lipschitz constant $C>0$. For $i=1,2$, let $\left(Y^{i}, Z^{i}, k^{i}, A^{i}\right)$ be the solution of the reflected BSDE with driver $f^{i}$, terminal time $T$, obstacle $\left(\eta_{t}^{i}\right)$, and terminal condition $\xi^{i}$. For $s \in[0, T]$, let $\bar{Y}_{s}:=Y_{s}^{1}-Y_{s}^{2}, \bar{\eta}_{s}:=\eta_{s}^{1}-\eta_{s}^{2}, \bar{\xi}:=\xi^{1}-\xi^{2}$ and $\bar{f}(s):=f^{1}\left(s, Y_{s}^{2}, Z_{s}^{2}, k_{s}^{2}\right)-f\left(s, Y_{s}^{2}, Z_{s}^{2}, k_{s}^{2}\right)$. Then, we have

$$
\|\bar{Y}\|_{\mathcal{S}^{2}}^{2} \leq K\left(\mathbb{E}\left[\bar{\xi}^{2}\right]+\mathbb{E}\left[\int_{0}^{T} \bar{f}^{2}(s) d s\right]\right)+\phi\left\|\sup _{0 \leq s<T}\left|\bar{\eta}_{s}\right|\right\|_{L^{2}},
$$

where the constant $K$ is universal, that is, depends only on the Lipschitz constant $C$ and $T$, and where the constant $\phi$ depends only on $C, T,\left\|\eta^{i}\right\|_{\mathcal{S}^{2}},\left\|\xi^{i}\right\|_{L^{2}}$, and $\left\|f^{i}(s, 0,0,0)\right\|_{\mathbb{H}^{2}}, i=1,2$.

Proof of Proposition 13. Let $n \in \mathbb{N}$. We apply (3.11) with $f^{1}=f \mathbf{1}_{t \leq \theta^{n}}, f^{2}=$ $f \mathbf{1}_{t \leq \theta}, \xi^{1}=\xi^{n}, \xi^{2}=\xi, \eta_{t}^{1}=\eta_{t} \mathbf{1}_{t<\theta^{n}}+\xi^{n} \mathbf{1}_{\theta^{n} \leq t<T}$, and $\eta_{t}^{2}=\eta_{t} \mathbf{1}_{t<\theta}+\eta_{\theta} \mathbf{1}_{\theta \leq t<\theta^{n}}+$ $\xi \mathbf{1}_{\theta^{n} \leq t<T}$. We have $Y_{\text {. }}^{1}=Y_{\text {., } \theta^{n}}\left(\xi^{n}\right)$ a.s. Moreover, since by assumption $\eta_{\theta} \leq \xi$ a.s., we have $Y_{.}^{2}=Y_{., \theta}(\xi)$ a.s. Note that $\left(Y_{t}^{2}, Z_{t}^{2}, k_{t}^{2}\right)=(\xi, 0,0)$ a.s. on $\{t \geq \theta\}$. We thus obtain

$$
\begin{aligned}
\left|Y_{0, \theta^{n}}\left(\xi^{n}\right)-Y_{0, \theta}(\xi)\right|^{2} \leq & K\left(\mathbb{E}\left[\left(\xi^{n}-\xi\right)^{2}\right]+\mathbb{E}\left[\int_{\theta}^{\theta^{n}} f^{2}(s, \xi, 0,0) d s\right]\right) \\
& +\phi\left\|\sup _{\theta \leq s<\theta^{n}}\left|\eta_{s}-\eta_{\theta}\right|\right\|_{L^{2}},
\end{aligned}
$$

where the constant $K$ depends only on the Lipschitz constant $C$ of $f$ and the terminal time $T$, and where the constant $\phi$ depends only on $C, T,\|\eta\|_{\mathcal{S}^{2}}, \sup _{n}\left\|\xi^{n}\right\|_{L^{2}}$, and $\|f(s, 0,0,0)\|_{\mathbb{H}^{2}}$. Since the obstacle $\left(\eta_{t}\right)$ is right-continuous and $\theta^{n} \downarrow \theta$ a.s., we have $\lim _{n \rightarrow+\infty}\left\|\sup _{\theta \leq s \leq \theta^{n}}\left|\eta_{s}-\eta_{\theta}\right|\right\|_{L^{2}}=0$. The right member of (3.12) thus tends to 0 as $n$ tends to $+\infty$. The result follows.

Remark 15. Compared with the case of nonreflected BSDEs (see [21, Proposition A.6]), there is an extra difficulty due to the presence of the obstacle (and the variation of the terminal time). The additional assumption (3.10) on the obstacle is here required to obtain the result.

Using Proposition 13, we derive a Fatou lemma in the reflected case, where the limit involves both terminal condition and terminal time.

Proposition 16 (a Fatou lemma for reflected BSDEs). Let $T>0$. Let $\left(\eta_{t}\right)$ be an RCLL process in $\mathcal{S}^{2}$. Let $f$ be a Lipschitz driver satisfying Assumption 12. Let $\left(\theta^{n}\right)_{n \in \mathbb{N}}$ be a nonincreasing sequence of stopping times in $\mathcal{T}$, converging a.s. to $\theta \in \mathcal{T}$ as $n$ tends to $\infty$. Let $\left(\xi^{n}\right)_{n \in \mathbb{N}}$ be a sequence of random variables such that $\mathbb{E}\left[\sup _{n}\left(\xi^{n}\right)^{2}\right]<+\infty$, and for each $n, \xi^{n}$ is $\mathcal{F}_{\theta^{n}}$-measurable.

Let $Y_{., \theta^{n}}\left(\xi^{n}\right) ; Y_{., \theta}\left(\liminf _{n \rightarrow+\infty} \xi^{n}\right)$ and $Y_{., \theta}\left(\limsup _{n \rightarrow+\infty} \xi^{n}\right)$ be the solution(s) of the reflected $B S D E(s)$ associated with driver $f$, obstacle $\left(\eta_{s}\right)_{s<\theta^{n}}$ (resp., $\left.\left(\eta_{s}\right)_{s<\theta}\right)$, terminal time $\theta^{n}$ (resp., $\left.\theta\right)$, and terminal condition $\xi^{n}$ (resp., $\liminf _{n \rightarrow+\infty} \xi^{n}$ and $\left.\lim \sup _{n \rightarrow+\infty} \xi^{n}\right)$. 
Suppose that

$$
\liminf _{n \rightarrow+\infty} \xi^{n} \geq \eta_{\theta} \quad\left(\text { resp., } \quad \limsup _{n \rightarrow+\infty} \xi^{n} \geq \eta_{\theta}\right) \quad \text { a.s. }
$$

Then $\quad Y_{0, \theta}\left(\liminf _{n \rightarrow+\infty} \xi^{n}\right) \leq \liminf _{n \rightarrow+\infty} Y_{0, \theta^{n}}\left(\xi^{n}\right)$

$$
\left(\text { resp., } \quad Y_{0, \theta}\left(\limsup _{n \rightarrow+\infty} \xi^{n}\right) \geq \limsup _{n \rightarrow+\infty} Y_{0, \theta^{n}}\left(\xi^{n}\right)\right) .
$$

When for each $n, \theta_{n}=\theta$ a.s., the result still holds without assumption (3.13).

Proof. We present only the proof of the first inequality, since the second one is obtained by similar arguments. For all $n$, we have by the monotonicity of reflected BSDEs with respect to terminal condition, $Y_{0, \theta^{n}}\left(\inf _{p \geq n} \xi^{p}\right) \leq Y_{0, \theta^{n}}\left(\xi^{n}\right)$. We derive that

$$
\liminf _{n \rightarrow+\infty} Y_{0, \theta^{n}}\left(\xi^{n}\right) \geq \liminf _{n \rightarrow+\infty} Y_{0, \theta^{n}}\left(\inf _{p \geq n} \xi^{p}\right)=Y_{0, \theta}\left(\liminf _{n \rightarrow+\infty} \xi^{n}\right)
$$

where the last equality follows from assumption (3.13) together with Proposition 13.

3.4. A weak DPP. We will now provide a weak DPP that is both a (weak) sub- and super-optimality principle of dynamic programming, involving, respectively, the u.s.c. envelope $u^{*}$ and the l.s.c. envelope $u_{*}$ of the value function $u$, defined by

$$
u^{*}(t, x):=\limsup _{\left(t^{\prime}, x^{\prime}\right) \rightarrow(t, x)} u\left(t^{\prime}, x^{\prime}\right) ; \quad u_{*}(t, x):=\liminf _{\left(t^{\prime}, x^{\prime}\right) \rightarrow(t, x)} u\left(t^{\prime}, x^{\prime}\right) \quad \forall(t, x) \in[0, T] \times \mathbb{R} .
$$

We now define the maps $\bar{u}^{*}$ and $\bar{u}_{*}$ for each $(t, x) \in[0, T] \times \mathbb{R}$ by

$$
\bar{u}^{*}(t, x):=u^{*}(t, x) \mathbf{1}_{t<T}+g(x) \mathbf{1}_{t=T} ; \quad \bar{u}_{*}(t, x):=u_{*}(t, x) \mathbf{1}_{t<T}+g(x) \mathbf{1}_{t=T} .
$$

Note that the functions $\bar{u}^{*}$ and $\bar{u}_{*}$ are Borelian. We have $\bar{u}_{*} \leq u \leq \bar{u}^{*}$ and $\bar{u}_{*}(T,)=$. $u(T,)=.\bar{u}^{*}(T,)=.g($.$) . Note that \bar{u}^{*}$ (resp., $\bar{u}_{*}$ ) is not necessarily upper (resp., lower) semicontinuous on $[0, T] \times \mathbb{R}$, since the terminal reward $g$ is only Borelian.

To prove the weak DPP, we will use the splitting properties (Theorem 7), the existence of $\varepsilon$-optimal controls (Theorem 11), and the Fatou lemma for RBSDEs (Proposition 16).

THEOREM 17 (a weak DPP). The value function u satisfies the following weak suboptimality principle of dynamic programming: for each $t \in[0, T]$ and for each stopping time $\theta \in \mathcal{T}_{t}^{t}$, we have

$$
u(t, x) \leq \sup _{\alpha \in \mathcal{A}_{t}^{t}} \sup _{\tau \in \mathcal{T}_{t}^{t}} \mathcal{E}_{t, \theta \wedge \tau}^{\alpha, t, x}\left[h\left(\tau, X_{\tau}^{\alpha, t, x}\right) \mathbf{1}_{\tau<\theta}+\bar{u}^{*}\left(\theta, X_{\theta}^{\alpha, t, x}\right) \mathbf{1}_{\tau \geq \theta}\right] .
$$

Moreover, the following weak superoptimality principle of dynamic programming holds: for each $t \in[0, T]$ and for each stopping time $\theta \in \mathcal{T}_{t}^{t}$, we have

$$
u(t, x) \geq \sup _{\alpha \in \mathcal{A}_{t}^{t}} \sup _{\tau \in \mathcal{T}_{t}^{t}} \mathcal{E}_{t, \theta \wedge \tau}^{\alpha, t, x}\left[h\left(\tau, X_{\tau}^{\alpha, t, x}\right) \mathbf{1}_{\tau<\theta}+\bar{u}_{*}\left(\theta, X_{\theta}^{\alpha, t, x}\right) \mathbf{1}_{\tau \geq \theta}\right] .
$$

Remark 18. The proof given below also shows that this weak DPP still holds with $\theta$ replaced by $\theta^{\alpha}$ in inequalities (3.14) and (3.15), given a family of stopping times indexed by controls $\left\{\theta^{\alpha}, \alpha \in \mathcal{A}_{t}^{t}\right\}$. 
Note that no regularity condition is required on $g$ to ensure this weak DPP, even (3.15). This is not the case in the literature even for classical expectation (see [8], [7], [4]). Moreover, our DPPs are stronger than those given in these papers, where inequality (3.14) (resp., (3.15)) is established with $u^{*}$ (resp., $u_{*}$ ) instead of $\bar{u}^{*}$ (resp., $\left.\bar{u}_{*}\right)$. Now, $\bar{u}^{*} \leq u^{*}$ and $\bar{u}_{*} \geq u_{*}$.

Before giving the proof, we introduce the following notation. For each $\theta \in \mathcal{T}$ and each $\xi$ in $L^{2}\left(\mathcal{F}_{\theta}\right)$, we denote by $\left(Y_{., \theta}^{\alpha, t, x}(\xi), Z_{., \theta}^{\alpha, t, x}(\xi), k_{., \theta}^{\alpha, t, x}(\xi)\right)$ the unique solution in $\mathcal{S}^{2} \times \mathbb{H}^{2} \times \mathbb{H}_{\nu}^{2}$ of the reflected BSDE with driver $f^{\alpha, t, x} \mathbf{1}_{\{s \leq \theta\}}$, terminal time $T$, terminal condition $\xi$, and obstacle $h\left(r, X_{r}^{\alpha, t, x}\right) \mathbf{1}_{r<\theta}+\xi \mathbf{1}_{r \geq \theta}$.

Proof. By estimates for reflected BSDEs (see [14, Proposition 5.1]), the function $u$ has at most polynomial growth at infinity. Hence, the random variables $\bar{u}^{*}\left(\theta, X_{\theta}^{\alpha, t, x}\right)$ and $\bar{u}_{*}\left(\theta, X_{\theta}^{\alpha, t, x}\right)$ are square integrable. Without loss of generality, to simplify notation, we suppose that $t=0$.

We first show the second assertion (which is the most difficult), or equivalently:

$$
\sup _{\alpha \in \mathcal{A}} Y_{0, \theta}^{\alpha, 0, x}\left[\bar{u}_{*}\left(\theta, X_{\theta}^{\alpha, 0, x}\right)\right] \leq u(0, x) \quad \forall \theta \in \mathcal{T} .
$$

Let $\theta \in \mathcal{T}$. For each $n \in \mathbb{N}$, we define

$$
\theta^{n}:=\sum_{k=0}^{2^{n}-1} t_{k} \mathbf{1}_{A_{k}}+T \mathbf{1}_{\theta=T},
$$

where $t_{k}:=\frac{(k+1) T}{2^{n}}$ and $A_{k}:=\left\{\frac{k T}{2^{n}} \leq \theta<\frac{(k+1) T}{2^{n}}\right\}$. Note that $\theta^{n} \in \mathcal{T}$ and $\theta^{n} \downarrow \theta$.

On $\{\theta=T\}$ we have $\theta^{n}=T$ for each $n$. We thus get $\bar{u}_{*}\left(\theta^{n}, X_{\theta^{n}}^{\alpha, 0, x}\right)=\bar{u}_{*}\left(\theta, X_{\theta}^{\alpha, 0, x}\right)$ for each $n$ on $\{\theta=T\}$. Moreover, on $\{\theta<T\}$, the lower semicontinuity of $\bar{u}_{*}$ on $\left[0, T\left[\times \mathbb{R}\right.\right.$ together with the right continuity of the process $X^{\alpha, 0, x}$ implies that

$$
\bar{u}_{*}\left(\theta, X_{\theta}^{\alpha, 0, x}\right) \leq \liminf _{n \rightarrow+\infty} \bar{u}_{*}\left(\theta^{n}, X_{\theta^{n}}^{\alpha, 0, x}\right) \quad \text { a.s. }
$$

Hence, by the comparison theorem for reflected BSDEs, we get

$$
Y_{0, \theta}^{\alpha, 0, x}\left[\bar{u}_{*}\left(\theta, X_{\theta}^{\alpha, 0, x}\right)\right] \leq Y_{0, \theta}^{\alpha, 0, x}\left[\liminf _{n \rightarrow+\infty} \bar{u}_{*}\left(\theta^{n}, X_{\theta^{n}}^{\alpha, 0, x}\right)\right] .
$$

On $\{\theta<T\}$, we have

$$
\begin{aligned}
\lim \inf _{n \rightarrow \infty} \bar{u}_{*}\left(\theta^{n}, X_{\theta^{n}}^{\alpha, 0, x}\right) & \geq \lim \inf _{n \rightarrow \infty} \bar{h}\left(\theta^{n}, X_{\theta^{n}}^{\alpha, 0, x}\right) \\
& =\lim _{n \rightarrow \infty} h\left(\theta^{n}, X_{\theta^{n}}^{\alpha, 0, x}\right)=h\left(\theta, X_{\theta}^{\alpha, 0, x}\right) \quad \text { a.s. }
\end{aligned}
$$

by the regularity properties of $h$ on $\left[0, T\left[\times \mathbb{R}\right.\right.$. On $\{\theta=T\}, \theta^{n}=T$ and

$$
\bar{u}_{*}\left(\theta^{n}, X_{\theta^{n}}^{\alpha, 0, x}\right)=\bar{u}_{*}\left(T, X_{T}^{\alpha, 0, x}\right)=g\left(X_{T}^{\alpha, 0, x}\right)=\bar{h}\left(T, X_{T}^{\alpha, 0, x}\right) .
$$

Hence, we have $\liminf _{n \rightarrow+\infty} \bar{u}_{*}\left(\theta^{n}, X_{\theta^{n}}^{\alpha, 0, x}\right) \geq \bar{h}\left(\theta, X_{\theta}^{\alpha, 0, x}\right)$ a.s. Condition (3.13) is thus satisfied with $\xi^{n}=\bar{u}_{*}\left(\theta^{n}, X_{\theta^{n}}^{\alpha, 0, x}\right)$ and $\xi_{t}=\bar{h}\left(t, X_{t}^{\alpha, 0, x}\right)$. We can thus apply the Fatou lemma for reflected BSDEs (Proposition 16). We thus get

$$
\begin{aligned}
Y_{0, \theta}^{\alpha, 0, x}\left[\bar{u}_{*}\left(\theta, X_{\theta}^{\alpha, 0, x}\right)\right] & \leq Y_{0, \theta}^{\alpha, 0, x}\left[\liminf _{n \rightarrow+\infty} \bar{u}_{*}\left(\theta^{n}, X_{\theta^{n}}^{\alpha, 0, x}\right)\right] \\
& \leq \liminf _{n \rightarrow \infty} Y_{0, \theta^{n}}^{\alpha, 0, x}\left[\bar{u}_{*}\left(\theta^{n}, X_{\theta^{n}}^{\alpha, 0, x}\right)\right] .
\end{aligned}
$$


Let $\varepsilon>0$. Fix $n \in \mathbb{N}$. For each $k<2^{n}-1$, let $\mathcal{A}_{t_{k}}$ be the set of the restrictions to $\left[t_{k}, T\right]$ of the controls $\alpha$ in $\mathcal{A}$. By Theorem 11, there exists a $P$-null set $\mathcal{N}$ (which depends on $n$ and $\varepsilon$ ) such that for each $k<2^{n}-1$, there exists an $\varepsilon$-optimal control control $\alpha^{n, \varepsilon, k}$ in $\mathcal{A}_{t_{k}}^{0}\left(=\mathcal{A}_{t_{k}}\right)$ for the control problem at time $t_{k}$ with initial condition $\eta=X_{t_{k}}^{\alpha, 0, x}$ that is satisfying the inequality

$$
u\left(t_{k}, X_{t_{k}, 0, x}^{\left.\left.\alpha{ }^{t_{k}} \omega\right)\right)} \leq u^{\alpha^{n, \varepsilon, k}\left({ }^{t_{k}} \omega, \cdot\right)}\left(t_{k}, X_{t_{k}}^{\alpha, 0, x}\left({ }^{t_{k}} \omega\right)\right)+\varepsilon\right.
$$

for each $\omega \in \mathcal{N}^{c}$. Using the definition of the maps $u^{\alpha^{n, \varepsilon, k}\left({ }_{k}{ }_{k} \omega, \cdot\right)}$ together with the splitting property for reflected BSDEs (3.7), we derive that there exists a $P$-null set $\mathcal{N}$ which contains the above one such that for each $\omega \in \mathcal{N}^{c}$ and for each $k<2^{n}-1$, we have

$$
\left.u^{\alpha^{n, \varepsilon, k}\left({ }^{t_{k}} \omega, \cdot\right)}\left(t_{k}, X_{t_{k}}^{\alpha, 0, x}\left({ }^{t_{k}} \omega\right)\right)=Y_{t_{k}, T}^{\alpha^{n, \varepsilon, k}\left({ }^{t_{k}} \omega, \cdot\right), t_{k}, X_{t_{k}}^{\alpha, 0, x}\left({ }^{t_{k}} \omega\right)}=Y_{t_{k}, T}^{\alpha^{n, \varepsilon, k}, t_{k}, X_{t_{k}}^{\alpha, 0, x}}{ }^{t_{k}} \omega\right) .
$$

Here, $Y_{., T}^{\alpha^{n, \varepsilon, k}, t_{k}, X_{t_{k}}^{\alpha, 0, x}}=Y_{., T}^{f^{\alpha^{n, \varepsilon, k}, t_{k}, X_{t_{k}}^{\alpha, 0, x}}}\left[\bar{h}\left(r, X_{r}^{\alpha^{n, \varepsilon, k}, t_{k}, X_{t_{k}}^{\alpha, 0, x}}\right)\right]$ denotes the solution of the reflected BSDE associated with terminal time $T$, obstacle $\left(\bar{h}\left(r, X_{r}^{\alpha^{n, \varepsilon, k}, t_{k}, X_{t_{k}}^{\alpha, 0, x}}\right)\right)_{t_{k} \leq r \leq T}$, and driver $f^{\alpha^{n, \varepsilon, k}, t_{k}, X_{t_{k}}^{\alpha, 0, x}}(r, y, z, k):=f\left(\alpha_{r}^{n, \varepsilon, k}, r, X_{r}^{\alpha, t_{k}, X_{t_{k}}^{\alpha, 0, x}}, y, z, k\right)$.

Set $\alpha_{s}^{n, \varepsilon}:=\sum_{k<2^{n}-1} \alpha_{s}^{n, \varepsilon, k} \mathbf{1}_{A_{k}}+\alpha_{s} \mathbf{1}_{\left\{\theta_{n}=T\right\}}$. Since for each $k, A_{k} \in \mathcal{F}_{t_{k}}$, there exists a $P$-null set $\mathcal{N}$ such that, on $\mathcal{N}^{c}$, for each $k<2^{n}-1$, we have the following equalities:

$$
\begin{aligned}
& Y_{t_{k}, T}^{\alpha^{n, \varepsilon, k}, t_{k}, X_{t_{k}}^{\alpha, 0, x}} \mathbf{1}_{A_{k}}=Y_{t_{k}, T}^{f^{\alpha^{n, \varepsilon, k}, t_{k}, X_{t_{k}}^{\alpha, 0, x}}} \mathbf{1}_{A_{k}}\left[\bar{h}\left(r, X_{r}^{\alpha^{n, \varepsilon, k}, t_{k}, X_{t_{k}}^{\alpha, 0, x}}\right) \mathbf{1}_{A_{k}}\right]
\end{aligned}
$$

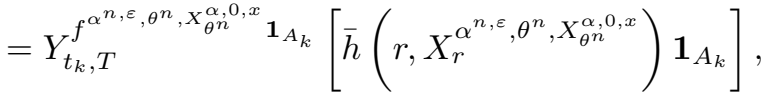

where, for a given driver $f, Y^{f \mathbf{1}_{A_{k}}}$ denotes the solution of the reflected BSDE associated with $f \mathbf{1}_{A_{k}}$. We thus get $Y_{t_{k}, T}^{\alpha^{n, \varepsilon, k}, t_{k}, X_{t_{k}}^{\alpha, 0, x}} \mathbf{1}_{A_{k}}=Y_{\theta^{n}, T}^{\alpha^{n, \varepsilon}, \theta^{n}, X_{\theta^{n}}^{\alpha, 0, x}} \mathbf{1}_{A_{k}}$ on $\mathcal{N}^{c}$. Using inequalities (3.19), we get

$$
\begin{gathered}
\bar{u}_{*}\left(\theta^{n}, X_{\theta^{n}}^{\alpha, 0, x}\right)=\sum_{0 \leq k<2^{n}-1} u_{*}\left(t_{k}, X_{t_{k}}^{\alpha, 0, x}\right) \mathbf{1}_{A_{k}}+g\left(X_{T}^{\alpha, t, x}\right) \mathbf{1}_{\left\{\theta_{n}=T\right\}} \\
\leq Y_{\theta^{n}, T}^{\alpha^{n, \varepsilon}, \theta^{n}, X_{\theta n}^{\alpha, 0, x}}+\varepsilon \text { on } \mathcal{N}^{c} .
\end{gathered}
$$

We set $\tilde{\alpha}_{s}^{n, \varepsilon}:=\alpha_{s} \mathbf{1}_{s<\theta^{n}}+\alpha_{s}^{n, \varepsilon} \mathbf{1}_{\theta^{n} \leq s \leq T}$. Note that $\tilde{\alpha}^{n, \varepsilon} \in \mathcal{A}$. Using the comparison theorem together with the estimates on reflected BSDEs (see [14]), we obtain

$$
Y_{0, \theta^{n}}^{\alpha, 0}\left[\bar{u}_{*}\left(\theta^{n}, X_{\theta^{n}}^{\alpha, 0, x}\right)\right] \leq Y_{0, \theta^{n}}^{\alpha, 0, x}\left[Y_{\theta^{n}, T}^{\alpha^{n, \varepsilon}, \theta^{n}, X_{\theta^{n}}^{\alpha, 0, x}}\right]+K \varepsilon=Y_{0, T}^{\tilde{\alpha}^{n, \varepsilon}, 0, x}+K \varepsilon,
$$

where the last equality follows from the flow property. Since $Y_{0, T}^{\tilde{\alpha}^{n, \varepsilon}, 0, x} \leq u(0, x)$, using (3.18), we get $Y_{0, \theta}^{\alpha, 0, x}\left[\bar{u}_{*}\left(\theta, X_{\theta}^{\alpha, 0, x}\right)\right] \leq Y_{0, \theta^{n}}^{\alpha, 0, x}\left[\bar{u}_{*}\left(\theta^{n}, X_{\theta^{n}}^{\alpha, 0, x}\right)\right] \leq u(0, x)+K \varepsilon$. Taking the supremum on $\alpha \in \mathcal{A}$ and letting $\varepsilon$ tend to 0 , we obtain inequality (3.16).

It remains to show the first assertion. It is sufficient to show that for each $\theta \in \mathcal{T}$,

$$
u(0, x) \leq \sup _{\alpha \in \mathcal{A}} Y_{0, \theta}^{\alpha, 0, x}\left[\bar{u}^{*}\left(\theta, X_{\theta}^{\alpha, 0, x}\right)\right] .
$$


Let $\theta \in \mathcal{T}$. Let $\alpha \in \mathcal{A}$. As above, we approximate $\theta$ by the sequence of stopping times $\left(\theta^{n}\right)_{n \in \mathbb{N}}$ defined above. Let $n \in \mathbb{N}$. By applying the flow property for reflected BSDEs, we get $Y_{0, T}^{\alpha, 0, x}=Y_{0, \theta^{n}}^{\alpha, 0, x}\left[Y_{\theta^{n}, T}^{\alpha, \theta^{n}, X_{\theta^{n}}^{\alpha, 0, x}}\right]$. By similar arguments as in the proof of the superoptimality principle (but without using the existence of $\varepsilon$-optimal controls), we derive that $Y_{\theta^{n}, T}^{\alpha, \theta^{n}, X_{\theta^{n}}^{\alpha, 0, x}} \leq \bar{u}^{*}\left(\theta^{n}, X_{\theta^{n}}^{\alpha, 0, x}\right)$ a.s. By the comparison theorem for reflected BSDEs, it follows that

$$
Y_{0, T}^{\alpha, 0, x}=Y_{0, \theta^{n}}^{\alpha, 0, x}\left[Y_{\theta^{n}, T}^{\alpha, \theta^{n}, X_{\theta^{n}}^{\alpha, 0, x}}\right] \leq Y_{0, \theta^{n}}^{\alpha, 0, x}\left[\bar{u}^{*}\left(\theta^{n}, X_{\theta^{n}}^{\alpha, 0, x}\right)\right] .
$$

Using the Fatou lemma for reflected BSDEs (Proposition 16), we get

$$
Y_{0, T}^{\alpha, 0, x} \leq \lim \sup _{n \rightarrow \infty} Y_{0, \theta^{n}}^{\alpha, 0, x}\left[\bar{u}^{*}\left(\theta^{n}, X_{\theta^{n}}^{\alpha, 0, x}\right)\right] \leq Y_{0, \theta}^{\alpha, 0, x}\left[\lim \sup _{n \rightarrow \infty} \bar{u}^{*}\left(\theta^{n}, X_{\theta^{n}}^{\alpha, 0, x}\right)\right] .
$$

Using the upper semicontinuity property of $\bar{u}^{*}$ on $\left[0, T\left[\times \mathbb{R}\right.\right.$ and $\bar{u}^{*}(T, x)=g(x)$, we obtain

$$
Y_{0, T}^{\alpha, 0, x} \leq Y_{0, \theta}^{\alpha, 0, x}\left[\lim \sup _{n \rightarrow \infty} \bar{u}^{*}\left(\theta^{n}, X_{\theta^{n}}^{\alpha, 0, x}\right)\right] \leq Y_{0, \theta}^{\alpha, 0, x}\left[\bar{u}^{*}\left(\theta, X_{\theta}^{\alpha, 0, x}\right)\right] .
$$

Since $\alpha \in \mathcal{A}$ is arbitrary, we get inequality (3.20), which completes the proof.

\section{Nonlinear HJB variational inequalities.}

4.1. Some extensions of comparison theorems for BSDEs and reflected BSDEs. We provide two results which will be used to prove that the value function $u$, defined by (2.6), is a weak viscosity solution of some nonlinear HJB variational inequalities (see Theorem 22). We first show a slight extension of the comparison theorem for BSDEs given in [21], from which we derive a comparison result between a BSDE and a reflected BSDE.

Lemma 19. Let $t_{0} \in[0, T]$ and let $\theta \in \mathcal{T}_{t_{0}}$. Let $\xi_{1}$ and $\xi_{2} \in L^{2}\left(\mathcal{F}_{\theta}\right)$. Let $f_{1}$ be a driver. Let $f_{2}$ be a Lipschitz driver with Lipschitz constant $C>0$, satisfying Assumption 12. For $i=1,2$, let $\left(X_{t}^{i}, \pi_{t}^{i}, l_{t}^{i}\right)$ be a solution in $\mathcal{S}^{2} \times \mathbb{H}^{2} \times \mathbb{H}_{\nu}^{2}$ of the $B S D E$ associated with driver $f_{i}$, terminal time $\theta$, and terminal condition $\xi_{i}$. Suppose that

$f_{1}\left(t, X_{t}^{1}, \pi_{t}^{1}, l_{t}^{1}\right) \geq f_{2}\left(t, X_{t}^{1}, \pi_{t}^{1}, l_{t}^{1}\right) \quad t_{0} \leq t \leq \theta, d t \otimes d P$ a.s., $\quad$ and $\quad \xi_{1} \geq \xi_{2}+\varepsilon$ a.s., where $\varepsilon$ is a real constant. Then, for each $t \in\left[t_{0}, \theta\right]$, we have $X_{t}^{1} \geq X_{t}^{2}+\varepsilon e^{-C T}$ a.s.

Proof. From inequality (4.22) in the proof of the comparison theorem in [21], we derive that $X_{t_{0}}^{1}-X_{t_{0}}^{2} \geq e^{-C T} \mathbb{E}\left[H_{t_{0}, \theta} \varepsilon \mid \mathcal{F}_{t_{0}}\right]$ a.s., where $C$ is the Lipschitz constant of $f_{2}$, and $\left(H_{t_{0}, s}\right)_{s \in\left[t_{0}, T\right]}$ is the nonnegative martingale satisfying $d H_{t_{0}, s}=$ $H_{t_{0}, s^{-}}\left[\beta_{s} d W_{s}+\int_{\mathbf{E}} \gamma_{s}(u) \tilde{N}(d s, d u)\right]$ with $H_{t_{0}, t_{0}}=1,\left(\beta_{s}\right)$ being a predictable process bounded by $C$. The result follows.

Proposition 20 (a comparison result between a BSDE and a reflected BSDE). Let $t_{0} \in[0, T]$ and let $\theta \in \mathcal{T}_{t_{0}}$. Let $\xi_{1} \in L^{2}\left(\mathcal{F}_{\theta}\right)$ and let $f_{1}$ be a driver. Let $\left(X_{t}^{1}, \pi_{t}^{1}, l_{t}^{1}\right)$ be a solution of the BSDE associated with $f_{1}$, terminal time $\theta$, and terminal condition $\xi^{1}$. Let $\left(\xi_{t}^{2}\right) \in \mathcal{S}^{2}$ and let $f_{2}$ be a Lipschitz driver with Lipschitz constant $C>0$ which satisfies Assumption 12. Let $\left(Y_{t}^{2}\right)$ be the solution of the reflected BSDE associated 
with $f_{2}$, terminal time $\theta$, and obstacle $\left(\xi_{t}^{2}\right)$. Suppose that

$$
\begin{array}{r}
f_{1}\left(t, X_{t}^{1}, \pi_{t}^{1}, l_{t}^{1}\right) \geq f_{2}\left(t, X_{t}^{1}, \pi_{t}^{1}, l_{t}^{1}\right), \quad t_{0} \leq t \leq \theta, d t \otimes d P-a . s . \\
\quad \text { and } X_{t}^{1} \geq \xi_{t}^{2}+\varepsilon, t_{0} \leq t \leq \theta \text { a.s. }
\end{array}
$$

Then, we have $X_{t}^{1} \geq Y_{t}^{2}+\varepsilon e^{-C T}, t_{0} \leq t \leq \theta$ a.s.

Proof. Let $t \in\left[t_{0}, \theta\right]$. By the characterization of the solution of the RBSDE as the value function of an optimal stopping problem (see Theorem 3.2 in [21]), $Y_{t}^{2}=$ ess $\sup _{\tau \in \mathcal{T}_{[t, \theta]}} \mathcal{E}_{t, \tau}^{f^{2}}\left(\xi_{\tau}^{2}\right)$. By Lemma 19, for each $\tau \in \mathcal{T}_{[t, \theta]}, X_{t}^{1} \geq \mathcal{E}_{t, \tau}^{f^{2}}\left(\xi_{\tau}^{2}\right)+e^{-C T} \varepsilon$. Taking the supremum over $\tau \in \mathcal{T}_{[t, \theta]}$, the result follows.

4.2. Links between the mixed control problem and HJB equation. We introduce the following HJB variational inequality (HJBVI):

$$
\left\{\begin{array}{l}
\min (u(t, x)-h(t, x) \\
\inf _{\alpha \in \mathbf{A}}\left(-\frac{\partial u}{\partial t}(t, x)-L^{\alpha} u(t, x)-f\left(\alpha, t, x, u(t, x),\left(\sigma \frac{\partial u}{\partial x}\right)(t, x), B^{\alpha} u(t, x)\right)\right) \\
\quad=0,(t, x) \in[0, T) \times \mathbb{R} \\
u(T, x)=g(x), x \in \mathbb{R},
\end{array}\right.
$$

where $L^{\alpha}:=A^{\alpha}+K^{\alpha}$, and for $\phi \in C^{2}(\mathbb{R})$,

- $A^{\alpha} \phi(x):=\frac{1}{2} \sigma^{2}(x, \alpha) \frac{\partial^{2} \phi}{\partial x^{2}}(x)+b(x, \alpha) \frac{\partial \phi}{\partial x}(x)$ and $B^{\alpha} \phi(x):=\phi(x+\beta(x, \alpha, \cdot))-$ $\phi(x)$,

- $K^{\alpha} \phi(x):=\int_{\mathbf{E}}\left(\phi(x+\beta(x, \alpha, e))-\phi(x)-\frac{\partial \phi}{\partial x}(x) \beta(x, \alpha, e)\right) \nu(d e)$.

Definition 21. A function $u$ is said to be a viscosity subsolution of (4.2) if it is u.s.c. on $[0, T] \times \mathbb{R}$, and if for any point $\left(t_{0}, x_{0}\right) \in[0, T[\times \mathbb{R}$ and for any $\phi \in$ $C^{1,2}([0, T] \times \mathbb{R})$ such that $\phi\left(t_{0}, x_{0}\right)=u\left(t_{0}, x_{0}\right)$ and $\phi-u$ attains its minimum at $\left(t_{0}, x_{0}\right)$, we have

$$
\begin{aligned}
\min \left(u\left(t_{0}, x_{0}\right)-h\left(t_{0}, x_{0}\right),\right. & \\
\inf _{\alpha \in \mathbf{A}}( & -\frac{\partial \phi}{\partial t}\left(t_{0}, x_{0}\right)-L^{\alpha} \phi\left(t_{0}, x_{0}\right) \\
& \left.-f\left(\alpha, t_{0}, x_{0}, u\left(t_{0}, x_{0}\right),\left(\sigma \frac{\partial \phi}{\partial x}\right)\left(t_{0}, x_{0}\right), B^{\alpha} \phi\left(t_{0}, x_{0}\right)\right)\right) \leq 0 .
\end{aligned}
$$

In other words, if $u\left(t_{0}, x_{0}\right)>h\left(t_{0}, x_{0}\right)$, then

$$
\begin{aligned}
\inf _{\alpha \in \mathbf{A}}( & -\frac{\partial \phi}{\partial t}\left(t_{0}, x_{0}\right)-L^{\alpha} \phi\left(t_{0}, x_{0}\right) \\
& \left.-f\left(\alpha, t_{0}, x_{0}, u\left(t_{0}, x_{0}\right),\left(\sigma \frac{\partial \phi}{\partial x}\right)\left(t_{0}, x_{0}\right), B^{\alpha} \phi\left(t_{0}, x_{0}\right)\right)\right) \leq 0
\end{aligned}
$$

A function $u$ is said to be a viscosity supersolution of (4.2) if it is l.s.c. on $[0, T] \times \mathbb{R}$, and if for any point $\left(t_{0}, x_{0}\right) \in\left[0, T\left[\times \mathbb{R}\right.\right.$ and any $\phi \in C^{1,2}([0, T] \times \mathbb{R})$ such 
that $\phi\left(t_{0}, x_{0}\right)=u\left(t_{0}, x_{0}\right)$ and $\phi-u$ attains its maximum at $\left(t_{0}, x_{0}\right)$, we have

$$
\begin{aligned}
\min \left(u\left(t_{0}, x_{0}\right)-h\left(t_{0}, x_{0}\right)\right. & \\
\inf _{\alpha \in \mathbf{A}}( & -\frac{\partial}{\partial t} \phi\left(t_{0}, x_{0}\right)-L^{\alpha} \phi\left(t_{0}, x_{0}\right) \\
& \left.-f\left(\alpha, t_{0}, x_{0}, u\left(t_{0}, x_{0}\right),\left(\sigma \frac{\partial \phi}{\partial x}\right)\left(t_{0}, x_{0}\right), B^{\alpha} \phi\left(t_{0}, x_{0}\right)\right)\right) \geq 0
\end{aligned}
$$

In other words, we have both $u\left(t_{0}, x_{0}\right) \geq h\left(t_{0}, x_{0}\right)$ and

$$
\begin{aligned}
\inf _{\alpha \in \mathbf{A}}( & -\frac{\partial \phi}{\partial t}\left(t_{0}, x_{0}\right)-L^{\alpha} \phi\left(t_{0}, x_{0}\right) \\
& \left.-f\left(\alpha, t_{0}, x_{0}, u\left(t_{0}, x_{0}\right),\left(\sigma \frac{\partial \phi}{\partial x}\right)\left(t_{0}, x_{0}\right), B^{\alpha} \phi\left(t_{0}, x_{0}\right)\right)\right) \geq 0
\end{aligned}
$$

Using the weak DPP given in Theorem 17 and Proposition 20, we now prove that the value function of our problem is a weak viscosity solution of the above HJBVI.

THEOREM 22. The value function $u$, defined by (2.6), is a weak viscosity solution of the HJBVI (4.2), in the sense that its u.s.c. envelope $u^{*}$ is a viscosity subsolution of (4.2) and its l.s.c. envelope $u_{*}$ is a viscosity supersolution of (4.2) (with terminal condition $u(T, x)=g(x))$.

Proof. We first prove that $u^{*}$ is a subsolution of $(4.2)$. Let $\left(t_{0}, x_{0}\right) \in[0, T[\times \mathbb{R}$ and $\phi \in C^{1,2}([0, T] \times \mathbb{R})$ be such that $\phi\left(t_{0}, x_{0}\right)=u^{*}\left(t_{0}, x_{0}\right)$ and $\phi(t, x) \geq u^{*}(t, x)$ for all $(t, x) \in[0, T] \times \mathbb{R}$. Without loss of generality, we can suppose that the minimum of $u^{*}-\phi$ attained at $\left(t_{0}, x_{0}\right)$ is strict. Suppose for contradiction that $u^{*}\left(t_{0}, x_{0}\right)>h\left(t_{0}, x_{0}\right)$ and that

$$
\begin{aligned}
\inf _{\alpha \in \mathbf{A}}( & -\frac{\partial}{\partial t} \phi\left(t_{0}, x_{0}\right)-L^{\alpha} \phi\left(t_{0}, x_{0}\right) \\
& \left.-f\left(\alpha, t_{0}, x_{0}, \phi\left(t_{0}, x_{0}\right),\left(\sigma \frac{\partial \phi}{\partial x}\right)\left(t_{0}, x_{0}\right), B^{\alpha} \phi\left(t_{0}, x_{0}\right)\right)\right)>0 .
\end{aligned}
$$

By uniform continuity of $K^{\alpha} \phi$ and $B^{\alpha} \phi:[0, T] \times \mathbb{R} \rightarrow L_{\nu}^{2}$ with respect to $\alpha$, we can suppose that there exists $\epsilon>0, \eta_{\epsilon}>0$ such that for all $(t, x)$ such that $t_{0} \leq t \leq$ $t_{0}+\eta_{\epsilon}<T$ and $\left|x-x_{0}\right| \leq \eta_{\epsilon}$, we have $\phi(t, x) \geq h(t, x)+\epsilon$ and

$$
-\frac{\partial}{\partial t} \phi(t, x)-L^{\alpha} \phi(t, x)-f\left(\alpha, t, x, \phi(t, x),\left(\sigma \frac{\partial \phi}{\partial x}\right)(t, x), B^{\alpha} \phi(t, x)\right) \geq \epsilon \forall \alpha \in \mathbf{A} .
$$

We denote by $B_{\eta_{\varepsilon}}\left(t_{0}, x_{0}\right)$ the ball of radius $\eta_{\varepsilon}$ and center $\left(t_{0}, x_{0}\right)$. By definition of $u^{*}$, there exists a sequence $\left(t_{n}, x_{n}\right)_{n}$ in $B_{\eta_{\varepsilon}}\left(t_{0}, x_{0}\right)$, such that $\left(t_{n}, x_{n}, u\left(t_{n}, x_{n}\right)\right) \rightarrow$ $\left(t_{0}, x_{0}, u^{*}\left(t_{0}, x_{0}\right)\right)$.

Fix $n \in \mathbb{N}$. Let $\alpha$ be an arbitrary control of $\mathcal{A}_{t_{n}}^{t_{n}}$ and $X^{\alpha, t_{n}, x_{n}}$ the associated state process.

We define the stopping time $\theta^{\alpha, n}$ as

$$
\theta^{\alpha, n}:=\left(t_{0}+\eta_{\epsilon}\right) \wedge \inf \left\{s \geq t_{n},\left|X_{s}^{\alpha, t_{n}, x_{n}}-x_{0}\right| \geq \eta_{\epsilon}\right\}
$$


Let $\psi^{\alpha}(s, x):=\frac{\partial}{\partial s} \phi(s, x)+L^{\alpha} \phi(s, x)$. Applying Itô's lemma to $\phi\left(t, X_{t}^{\alpha, t_{n}, x_{n}}\right)$, we derive that

$$
\left(\phi\left(s, X_{s}^{\alpha, t_{n}, x_{n}}\right),\left(\sigma \frac{\partial \phi}{\partial x}\right)\left(s, X_{s}^{\alpha, t_{n}, x_{n}}\right), B^{\alpha_{s}} \phi\left(s, X_{s^{-}}^{\alpha, t_{n}, x_{n}}\right) ; s \in\left[t_{n}, \theta^{\alpha, n}\right]\right)
$$

is the solution of the BSDE associated with the driver process $-\psi^{\alpha_{s}}\left(s, X_{s}^{\alpha, t_{n}, x_{n}}\right)$, terminal time $\theta^{\alpha, n}$, and terminal value $\phi\left(\theta^{\alpha, n}, X_{\theta^{\alpha, n}}^{\alpha, t_{n}, x_{n}}\right)$. By (4.5) and by definition of $\theta^{\alpha, n}$, we get

$$
\begin{aligned}
& -\psi^{\alpha_{s}}\left(s, X_{s}^{\alpha, t_{n}, x_{n}}\right) \\
\geq & f\left(\alpha_{s}, s, X_{s}^{\alpha, t_{n}, x_{n}}, \phi\left(s, X_{s}^{\alpha, t_{n}, x_{n}}\right),\left(\sigma \frac{\partial \phi}{\partial x}\right)\left(s, X_{s}^{\alpha, t_{n}, x_{n}}\right), B \phi\left(s, X_{s}^{\alpha, t_{n}, x_{n}}\right)\right)+\epsilon
\end{aligned}
$$

for each $s \in\left[t_{n}, \theta^{\alpha, n}\right]$. This inequality gives a relation between the drivers $-\psi^{\alpha_{s}}$ $\left(s, X_{s}^{\alpha, t_{n}, x_{n}}\right)$ and $f\left(\alpha_{s}, \cdot\right)$ of two BSDEs. Now, since the minimum $\left(t_{0}, x_{0}\right)$ is strict, there exists $\gamma_{\epsilon}$ such that

$$
u^{*}(t, x)-\phi(t, x) \leq-\gamma_{\epsilon} \text { on }[0, T] \times \mathbb{R} \backslash B_{\eta_{\epsilon}}\left(t_{0}, x_{0}\right) .
$$

We have

$$
\begin{aligned}
\phi\left(\theta^{\alpha, n} \wedge t, X_{\theta^{\alpha, n} \wedge t}^{\alpha, t_{n}, x_{n}}\right)= & \phi\left(t, X_{t}^{\alpha, t_{n}, x_{n}}\right) \mathbf{1}_{t<\theta^{\alpha, n}} \\
& +\phi\left(\theta^{\alpha, n}, X_{\theta^{\alpha, n}}^{\alpha, t_{n}, x_{n}}\right) \mathbf{1}_{t \geq \theta^{\alpha, n}}, \quad t_{n} \leq t \leq T \quad \text { a.s. }
\end{aligned}
$$

To simplify notation, set $\delta_{\varepsilon}:=\min \left(\epsilon, \gamma_{\epsilon}\right)$. Using (4.7) together with the definition of $\theta^{\alpha, n}$, we get

$$
\begin{aligned}
\phi\left(t, X_{t}^{\alpha, t_{n}, x_{n}}\right) \geq & \left(h\left(t, X_{t}^{\alpha, t_{n}, x_{n}}\right)+\delta_{\varepsilon}\right) \mathbf{1}_{t<\theta^{\alpha, n}} \\
& +\left(u^{*}\left(\theta^{\alpha, n}, X_{\theta^{\alpha, n}}^{\alpha, t_{n}, x_{n}}\right)+\delta_{\varepsilon}\right) \mathbf{1}_{t=\theta^{\alpha, n}}, \quad t_{n} \leq t \leq \theta^{\alpha, n} \quad \text { a.s. }
\end{aligned}
$$

This, together with inequality (4.6) on the drivers and the above comparison theorem between a BSDE and a reflected BSDE (see Proposition 20) leads to

$$
\phi\left(t_{n}, x_{n}\right) \geq Y_{t_{n}, \theta^{\alpha, n}}^{\alpha, t_{n}, x_{n}}\left[h\left(t, X_{t}^{\alpha, t_{n}, x_{n}}\right) \mathbf{1}_{t<\theta^{\alpha, n}}+u^{*}\left(\theta^{\alpha, n}, X_{\theta^{\alpha, n}}^{\alpha, t_{n}, x_{n}}\right) \mathbf{1}_{t=\theta^{\alpha}}\right]+\delta_{\varepsilon} K,
$$

where $K$ is a positive constant which only depends on $T$ and the Lipschitz constant of $f$.

Now, recall that $\left(t_{n}, x_{n}, u\left(t_{n}, x_{n}\right)\right) \rightarrow\left(t_{0}, x_{0}, u^{*}\left(t_{0}, x_{0}\right)\right)$ and $\phi$ is continuous with $\phi\left(t_{0}, x_{0}\right)=u^{*}\left(t_{0}, x_{0}\right)$. We can thus assume that $n$ is sufficiently large so that $\left|\phi\left(t_{n}, x_{n}\right)-u\left(t_{n}, x_{n}\right)\right| \leq \delta_{\varepsilon} K / 2$. Hence,

$$
u\left(t_{n}, x_{n}\right) \geq Y_{t_{n}, \theta^{\alpha, n}}^{\alpha, t_{n}, x_{n}}\left[h\left(t, X_{t}^{\alpha, t_{n}, x_{n}}\right) \mathbf{1}_{t<\theta^{\alpha, n}}+u^{*}\left(\theta^{\alpha, n}, X_{\theta^{\alpha, n}}^{\alpha, t_{n}, x_{n}}\right) \mathbf{1}_{t=\theta^{\alpha}}\right]+\delta_{\varepsilon} K / 2 .
$$

As this inequality holds for all $\alpha \in \mathcal{A}_{t_{n}}^{t_{n}}$ and since $u^{*} \geq \bar{u}^{*}$, we get a contradiction of the suboptimality principle of DPP (3.14) (see also Remark 18).

We now prove that $u_{*}$ is a viscosity supersolution of $(4.2)$. Let $\left(t_{0}, x_{0}\right) \in[0, T[\times \mathbb{R}$ and $\phi \in C^{1,2}([0, T] \times \mathbb{R})$ be such that $\phi\left(t_{0}, x_{0}\right)=u_{*}\left(t_{0}, x_{0}\right)$ and $\phi(t, x) \leq u_{*}(t, x)$ for all $(t, x) \in[0, T] \times \mathbb{R}$. Without loss of generality, we can suppose that the maximum is strict in $\left(t_{0}, x_{0}\right)$. Since the solution $\left(Y_{s}^{\alpha, t_{0}, x_{0}}\right)$ stays above the obstacle, for each $\alpha \in \mathcal{A}$, we have $u_{*}\left(t_{0}, x_{0}\right) \geq h\left(t_{0}, x_{0}\right)$. Our aim is to show that inequality (4.4) holds. 
Suppose for contradiction that this inequality does not hold.

By continuity, we can suppose that there exists $\alpha \in \mathbf{A}, \epsilon>0$, and $\eta_{\epsilon}>0$ such that for all $(t, x)$ with $t_{0} \leq t \leq t_{0}+\eta_{\epsilon}<T$ and $\left|x-x_{0}\right| \leq \eta_{\epsilon}$, we have

$$
-\frac{\partial}{\partial t} \phi(t, x)-L^{\alpha} \phi(t, x)-f\left(\alpha, t, x, \phi(t, x),\left(\sigma \frac{\partial \phi}{\partial x}\right)(t, x), B^{\alpha} \phi(t, x)\right) \leq-\epsilon .
$$

We denote by $B_{\eta_{\varepsilon}}\left(t_{0}, x_{0}\right)$ the ball of radius $\eta_{\varepsilon}$ and center $\left(t_{0}, x_{0}\right)$. Let $\left(t_{n}, x_{n}\right)_{n}$ be a sequence in $B_{\eta_{\varepsilon}}\left(t_{0}, x_{0}\right)$ such that $\left(t_{n}, x_{n}, u\left(t_{n}, x_{n}\right)\right) \rightarrow\left(t_{0}, x_{0}, u_{*}\left(t_{0}, x_{0}\right)\right)$. We introduce the state process $X^{\alpha, t_{n}, x_{n}}$ associated with the above constant control $\alpha$ and define the stopping time $\theta^{n}$ as

$$
\theta^{n}:=\left(t_{0}+\eta_{\epsilon}\right) \wedge \inf \left\{s \geq t_{n},\left|X_{s}^{\alpha, t_{n}, x_{n}}-x_{0}\right| \geq \eta_{\epsilon}\right\} .
$$

By Itô's formula, the process $\left(\phi\left(s, X_{s}^{\alpha, t_{n}, x_{n}}\right),\left(\sigma \frac{\partial \phi}{\partial x}\right)\left(s, X_{s}^{\alpha, t_{n}, x_{n}}\right), B^{\alpha} \phi\left(s, X_{s^{-}}^{\alpha, t_{n}, x_{n}}\right) ; s \in\right.$ $\left.\left[t_{n}, \theta^{n}\right]\right)$ is the solution of the BSDE associated with terminal time $\theta^{n}$, terminal value $\phi\left(\theta^{n}, X_{\theta^{n}}^{\alpha, t_{n}, x_{n}}\right)$, and driver $-\psi^{\alpha}\left(s, X_{s}^{\alpha, t_{n}, x_{n}}\right)$. The definition of the stopping time $\theta^{n}$ and inequality (4.8) lead to

$$
\begin{aligned}
& -\psi^{\alpha}\left(s, X_{s}^{\alpha, t_{n}, x_{n}}\right) \\
& \leq f\left(\alpha, s, X_{s}^{\alpha, t_{n}, x_{n}}, \phi\left(s, X_{s}^{\alpha, t_{n}, x_{n}}\right),\left(\sigma \frac{\partial \phi}{\partial x}\right)\left(s, X_{s}^{\alpha, t_{n}, x_{n}}\right), B^{\alpha} \phi\left(s, X_{s}^{\alpha, t_{n}, x_{n}}\right)\right)
\end{aligned}
$$

for $t_{n} \leq s \leq \theta^{n} d s \otimes d P$-a.s. Now, since the maximum $\left(t_{0}, x_{0}\right)$ is strict, there exists $\gamma_{\epsilon}$ (which depends on $\eta_{\epsilon}$ ) such that $u_{*}(t, x) \geq \phi(t, x)+\gamma_{\epsilon}$ on $[0, T] \times \mathbb{R} \backslash B_{\eta_{\epsilon}}\left(t_{0}, x_{0}\right)$ which implies $\phi\left(\theta^{n}, X_{\theta^{n}}^{\alpha, t_{n}, x_{n}}\right) \leq u_{*}\left(\theta^{n}, X_{\theta^{n}}^{\alpha, t_{n}, x_{n}}\right)-\gamma_{\epsilon}$. Hence, using inequality (4.9) on the drivers, together with the comparison theorem for BSDEs, we derive that

$$
\begin{aligned}
\phi\left(t_{n}, x_{n}\right) & =\mathcal{E}_{t_{n}, \psi^{n}}^{-\psi^{\alpha}}\left[\phi\left(\theta^{n}, X_{\theta^{n}}^{\alpha, t_{n}, x_{n}}\right)\right] \leq \mathcal{E}_{t_{n}, \theta^{n}}^{\alpha, t_{n}, x_{n}}\left[u_{*}\left(\theta^{n}, X_{\theta}^{\alpha, t_{n}, x_{n}}\right)-\gamma_{\epsilon}\right] \\
& \leq \mathcal{E}_{t_{n}, \theta^{n}}^{\alpha, t_{n}, x_{n}}\left[u_{*}\left(\theta^{n}, X_{\theta^{n}}^{\alpha, t_{n}, x_{n}}\right)\right]-\gamma_{\epsilon} K,
\end{aligned}
$$

where the second inequality follows from an extension of the comparison theorem (Lemma 19). We can assume that $n$ is sufficient large so that $\left|\phi\left(t_{n}, x_{n}\right)-u\left(t_{n}, x_{n}\right)\right| \leq$ $\delta_{\varepsilon} K / 2$. We thus get

$$
u\left(t_{n}, x_{n}\right) \leq \mathcal{E}_{t_{n}, \theta^{n}}^{\alpha, t_{n}, x_{n}}\left[u_{*}\left(\theta^{n}, X_{\theta^{n}}^{\alpha, t_{n}, x_{n}}\right)\right]-\gamma_{\epsilon} K / 2
$$

Since $u$ satisfies the superoptimality DPP (Theorem 17), we have $u\left(t_{n}, x_{n}\right) \geq \mathcal{E}_{t_{n}, \theta^{n}}^{\alpha, t_{n}, x_{n}}$ $\left[\bar{u}_{*}\left(\theta^{n}, X_{\theta^{n}}^{\alpha, t_{n}, x_{n}}\right)\right]$. Since $\bar{u}_{*} \geq u_{*}$, this inequality with (4.10) leads to a contradiction.

Remark 23. When $g$ is only Borelian, the weak solution of the HJB equation (4.2) is generally not unique, even in the deterministic case (as stressed in [2], [1], [3]).

Note that when $g$ is l.s.c., the value function $u$ of our problem can be shown to be the minimal (l.s.c.) viscosity supersolution of the HJB equation (4.2), with terminal value greater than $g$ (by using similar arguments as in the proof of [15, Theorem 6.5]). 
Note also that the paper [2] (see also [1]) provides a characterization of the u.s.c. envelope $u^{*}$ of the value function of the deterministic control problem $u(t, x):=$ $\sup _{\alpha \in \mathcal{A}_{t}} g\left(X_{T}^{t, x, \alpha}\right)$, which corresponds to our problem with $\sigma=f=0$ and no stopping times controls. More precisely, when $g$ is u.s.c., the map $u^{*}$ is characterized as the unique u.s.c. viscosity solution of the HJB equation (i.e., satisfies $u^{*}(T, x)=g(x)$ and the analogous of (4.3) but with an equality). The proof is based on PDEs arguments and deterministic control theory. An interesting further development of our paper (and of [8]) would be to study analogous properties in the stochastic case.

Appendix A. Appendix. We give here some measurability results which are used in section 3.2. We start by the proof of Proposition 9. To this purpose, we first provide the following lemma.

Lemma 24. Let $(\Omega, \mathcal{F}, P)$ be a probability space. Suppose that the Hilbert space $L^{2}:=L^{2}(\Omega, \mathcal{F}, P)$ equipped with the usual scalar product is separable. Let $F \in L^{2}$.

Consider a sequence of functions $\left(g_{n}\right)_{n \in \mathbb{N}}$ such that for each $n, g_{n}: \mathbb{R} \rightarrow \mathbb{R}$ is Borelian with $\left|g_{n}(x)\right| \leq C\left(1+|x|^{p}\right)$. Suppose that sequence $\left(g_{n}\right)_{n \in \mathbb{N}}$ converges pointwise.

Let $g$ be the limit, defined for each $x \in \mathbb{R}$ by $g(x):=\lim _{n \rightarrow+\infty} g_{n}(x)$.

Suppose also that for each $n \in \mathbb{N}$, the map $\psi^{g_{n}, F}$ (denoted also by $\psi^{g_{n}}$ ) defined by $\psi^{g_{n}}: L^{2 p} \cap L^{2} \rightarrow \mathbb{R} ; \xi \mapsto \mathbb{E}\left[g_{n}(\xi) F\right]$ is Borelian, $L^{2 p} \cap L^{2}$ being equipped with the $\sigma$-algebra induced by $\mathcal{B}\left(L^{2}\right)$.

Then, the map $\psi^{g}$ (denoted also by $\psi^{g, F}$ ) defined by

$$
\psi^{g}: L^{2 p} \cap L^{2} \rightarrow \mathbb{R} ; \xi \mapsto \mathbb{E}[g(\xi) F]
$$

is Borelian.

Proof. By the Lebesgue theorem, for each $\xi \in L^{2 p} \cap L^{2}$, we have $\psi^{g}(\xi)=$ $\mathbb{E}[g(\xi) F]=\lim _{n \rightarrow+\infty} \mathbb{E}\left[g_{n}(\xi) F\right]=\lim _{n \rightarrow+\infty} \psi^{g_{n}}(\xi)$. Since the pointwise limit of a sequence of $\mathbb{R}$-valued measurable maps is measurable, we derive that the map $\psi^{g}$ is Borelian.

Proof of Proposition 9. Since by assumption the Hilbert space $L^{2}$ is separable, there exists a countable orthonormal basis $\left\{e^{i}, i \in \mathbb{N}\right\}$ of $L^{2}$. For each $\xi \in L^{2 p} \cap L^{2}$, we have $\varphi^{g}(\xi)=g(\xi)=\sum_{i} \psi^{g, i}(\xi) e_{i}$ in $L^{2}$, where $\psi^{g, i}(\xi):=\mathbb{E}\left[g(\xi) e_{i}\right]$ for each $i \in \mathbb{N}$. Hence, in order to show the measurability of the map $\psi$, it is sufficient to show the measurability of the maps $\psi^{g, F}, F \in L^{2}$.

For this purpose, we introduce the set $\mathcal{H}$ of bounded Borelian functions $g: \mathbb{R} \rightarrow \mathbb{R}$ such that for each $F \in L^{2}$, the map $\psi^{g, F}$ is Borelian. Note that $\mathcal{H}$ is a vector space. Suppose we have shown that for all real numbers $a, b$ with $a<b, \mathbf{1}_{] a, b[} \in \mathcal{H}$. Then, by Lemma 24 together with a monotone class theorem, we derive that $\mathcal{H}$ is equal to the whole set of bounded Borelian functions. When $g$ is not bounded, the result follows by approximating $g$ by a sequence of bounded Borelian functions, and by using Lemma 24.

It remains to show that for all $a, b \in \mathbb{R}$ with $a<b$, we have $\mathbf{1}_{] a, b[} \in \mathcal{H}$. Since $\mathbf{1}_{] a, b[}$ is l.s.c., it follows that there exists a nondecreasing sequence $\left(g_{n}\right)_{n \in \mathbb{N}}$ of Lipschitz continuous functions (taking their values in $[0,1]$ ) such that for each $x \in \mathbb{R}, \mathbf{1}_{] a, b[}(x):=$ $\lim _{n \rightarrow+\infty} g_{n}(x)$. For each $n$, since $g_{n}$ is Lipschitz continuous, by using the CauchySchwarz inequality, one can derive that the map $\psi^{g_{n}}: L^{2} \rightarrow \mathbb{R} ; \xi \mapsto \mathbb{E}\left[g_{n}(\xi) F\right]$ is Lipschitz continuous for the norm $\|\cdot\|_{L^{2}}$, and hence Borelian. The result then follows from Lemma 24. 
We now state the following lemma, which is needed in the proof of Theorem 10.

Lemma 25. Let $t \in[0, T]$. The Hilbert space $L^{2}\left(\Omega, \mathcal{F}_{T}^{t}, P\right)$ (simply denoted by $\left.L_{t}^{2}\right)$ is separable. Moreover, the Hilbert space $\mathbb{H}_{t}^{2}$ is separable.

Proof. The proof is given for completeness. Recall first that, given a probabilistic space $(\Omega, \mathcal{B}, P)$, if $\mathcal{B}$ is countably generated, then $L^{2}(\Omega, \mathcal{B}, P)$ is separable (see, e.g., [11, Proposition 3.4.5]). In our case, $\Omega$ is separable, which implies that the Borelian $\sigma$-algebra $\mathcal{B}(\Omega)$ is countably generated. The space $L^{2}(\Omega, \mathcal{B}(\Omega), P)$ is thus separable. Now let $t$ be any real in $[0, T]$. We introduce $\mathbb{F}^{o, t}=\left(\mathcal{F}_{s}^{o, t}\right)_{s \geq t}$, the natural filtration of $W^{t}$ and $N^{t}$. By definition, $\mathbb{F}^{t}$ is the completed filtration of $\mathbb{F}^{o, t}$ (with respect to $\mathcal{B}(\Omega)$ and $P$ ). For each $\xi \in L_{t}^{2}=L^{2}\left(\Omega, \mathcal{F}_{T}^{t}, P\right.$ ), there exists an $\mathcal{F}_{T}^{o, t}$ measurable random variable $\xi^{\prime}$ such that $\xi=\xi^{\prime}, P$-a.s. Hence, $L_{t}^{2}$ can be identified with $L^{2}\left(\Omega, \mathcal{F}_{T}^{o, t}, P\right)$, which is separable because $\mathcal{F}_{T}^{o, t}=\left(T^{t}\right)^{-1}(\mathcal{B}(\Omega))$ is countably generated.

Now, denote by $\mathcal{P}^{o, t}$ the predictable $\sigma$-algebra associated with $\mathbb{F}^{o, t}$. For each $\mathcal{P}^{t}$-measurable process $\left(X_{s}\right)$, there exists a $\mathcal{P}^{o, t}$-measurable process $\left(X_{s}^{\prime}\right)$ indistinguishable of $\left(X_{s}\right)$ (see [13, IV, section 79] or [17, I, Proposition 1.1, p. 8]). Hence, the space $\mathbb{H}_{t}^{2}=L^{2}\left([t, T] \times \Omega, \mathcal{P}^{t}, d s \otimes d P\right)$ can be identified with the Hilbert space $L^{2}\left([t, T] \times \Omega, \mathcal{P}^{o, t}, d s \otimes d P\right)$. Since the paths are right-continuous, for every $r>t$, $\mathcal{F}_{r^{-}}^{o, t}=\sigma\left(\left\{\omega_{u}^{t}, u \in \mathbb{Q}\right.\right.$ and $\left.\left.t \leq u<r\right\}\right)$ and is thus countably generated. The predictable $\sigma$-algebra $\mathcal{P}^{o, t}$ is generated by the sets of the form $[r, T[\times H($ or $] r, T] \times H)$, where $r$ is rational with $r \geq t$, and $H$ belongs to $\mathcal{F}_{r^{-}}^{o, t}$. It follows that $\mathcal{P}^{o, t}$ is countably generated. Hence, $L^{2}\left([t, T] \times \Omega, \mathcal{P}^{o, t}, d s \otimes d P\right)$ is separable, which gives that $\mathbb{H}_{t}^{2}$ is separable.

Lemma 1.2 in [12] ensures the following property, which is used in the proof of Theorem 11.

Lemma 26 (a result of measure theory). Let $(X, \mathcal{F}, Q)$ be a probability space. Let $\mathcal{F}_{Q}$ be the completion $\sigma$-algebra of $\mathcal{F}$ with respect to $Q$ that is the class of sets of the form $B \cup M$ with $B \in \mathcal{F}$ and $M$ being a $Q$-null set that is a subset of a set $N$ belonging to $\mathcal{F}$ with $Q$-measure 0 . Let $E$ be a separable Hilbert space, equipped with its scalar product $\langle.,$.$\rangle , and its Borel \sigma$-algebra $\mathcal{B}(E)$.

Then, for each $\mathcal{F}_{Q}$-measurable map $f: X \rightarrow E$, there exists an $\mathcal{F}$-measurable map $f_{Q}$ such that $f_{Q}(x)=f(x)$ for $Q$-almost every $x$, in the sense that the set $\left\{x \in X, f_{Q}(x) \neq f(x)\right\}$ is included in a set belonging to $\mathcal{F}$ with $Q$-measure 0 .

$A$ result of classical analysis (used in the proof of Lemma 5). For each $n \in \mathbb{N}$, we consider the linear operator $P^{n}: L^{2}([0, T], d r) \rightarrow L^{2}([0, T], d r)$ defined for each $f \in$ $L^{2}([0, T], d r)$ by $P^{n}(f)(t):=n \sum_{i=1}^{n-1}\left(\int_{\frac{i-1) T}{n}}^{\frac{i T}{n}} f(r) d r\right) \mathbf{1}_{\left.\frac{i T}{n}, \frac{(i+1) T}{n}\right]}(t)$. By the CauchySchwarz inequality, we have that for each $\left.t \in] \frac{i T}{n}, \frac{(i+1) T}{n}\right], 1 \leq i \leq n-1, P^{n}(f)^{2}(t) \leq$ $n \int_{\frac{(i-1) T}{n}}^{\frac{i T}{n}} f^{2}(r) d r$. Hence,

$$
\left\|P_{n}(f)\right\|_{L_{T}^{2}} \leq\|f\|_{L_{T}^{2}} ; \quad\left\|P_{n}(f)-f\right\|_{L_{T}^{2}} \rightarrow 0, \text { when } n \rightarrow \infty .
$$

The above convergence clearly holds when $f$ is continuous, and the general case follows by using the uniform continuity of $f$ and the density of $\mathcal{C}([0, T])$ in $L_{T}^{2}$.

Acknowledgments. We are grateful to Guy Barles, Rainer Buckdahn, Halim Doss, and Nicole El Karoui for valuable discussions. We thank the anonymous referees for their helpful remarks. 


\section{REFERENCES}

[1] G. Barles, Discontinuous viscosity solutions of first-order Hamilton-Jacobi equations: A guided visit, Nonlinear Anal., 20 (1993), pp. 1123-1134.

[2] E.N. Barron and R. Jensen, Optimal control and semicontinuous viscosity solutions, Proc. Amer. Math. Soc., 113 (1991).

[3] G. Barles and B. Perthame, Discontinuous viscosity solutions of deterministic optimal control problems, in Proceedings of the 1986 25th IEEE Conference on Decision and Control, 1986.

[4] E. Bayraktar And S. YaO, A weak dynamic programming principle for zero-sum stochastic differential games with unbounded controls, SIAM J. Control Optim., 51 (2013), pp. 20362080.

[5] E. Bayraktar and S. YaO, Optimal stopping for non-linear expectations, Stochastic Process. Appl., 121 (2011), pp. 185-211 and 212-264.

[6] D. Bertsekas and S. Shreve, Stochastic Optimal Control: The Discrete Time Case, Academic Press, Orlando, FL, 1978.

[7] B. Bouchard AND M. Nutz, Weak dynamic programming for generalized state constraints, SIAM J. Control Optim., 50 (2012), pp. 3344-3373.

[8] B. Bouchard AND N. TouzI, Weak dynamic programming principle for viscosity solutions, SIAM J. Control Optim., 49 (2011), pp. 948-962.

[9] R. Buckdahn AND J. Li, Stochastic differential games and viscosity solutions of HamiltonJacobi-Bellman-Isaacs equations, SIAM J. Control Optim., 47 (2008), pp. 444-475.

[10] R. Buckdahn And T. Nie, Generalized Hamilton-Jacobi-Bellman Equations with Dirichlet Boundary and Stochastic Exit Time Optimal Control Problem, http://arxiv.org/pdf/1412. 0730v4.pdf, 2014.

[11] D. Cohn, Measure Theory, 2nd ed., Birkhäuser, Basel, 2013.

[12] H. Crauel, Random Probability Measures on Polish Spaces, Stoch. Monogr. 11, Taylor and Francis, London, 2002.

[13] C. Dellacherie and P.-A. Meyer, Probabilités et Potentiel, Hermann, Paris, 1975, MR0488194.

[14] R. Dumitrescu, M.C. Quenez, And A. Sulem, Optimal stopping for dynamic risk measures with jumps and obstacle problems, J. Optim. Theory Appl., 167 (2015), pp. 219-242.

[15] R. Dumitrescu, M.C. Quenez, And A. Sulem, Mixed Generalized Dynkin Games and Stochastic Control in a Markovian Framework, http://arxiv.org/abs/1508.02742, 2015.

[16] N. El Karoui, C. Kapoudjian, E. Pardoux, S. Peng, and M.-C. Quenez, Reflected solutions of backward SDE's, and related obstacle problems for PDE's, Ann. Probab., 25 (1997), pp. $702-737$.

[17] J. JACOD, Calcul Stochastique et Problèmes de Martingales, Springer, Berlin, 1979.

[18] J. Li AND S. Peng, Stochastic optimization theory of backward stochastic differential equations with jumps and viscosity solutions of Hamilton-Jacobi-Bellman equations, Nonlinear Anal., 70 (2009), pp. 1776-1796.

[19] P.L. Lions And P.E. Souganidis, Differential games, optimal control and directional derivatives of viscosity solutions of Bellman's and Isaacs' equations, SIAM J. Control Optim., 23 (1985), pp. 566-583.

[20] S. Peng, A generalized dynamic programming principle and Hamilton-Jacobi-Bellman equation, Stoch. Stoch. Rep., 38 (1992), pp. 119-134.

[21] M.-C. Quenez And A. Sulem, BSDEs with jumps, optimization and applications to dynamic risk measures, Stochastic Process. Appl., 123 (2013), pp. 3328-3357.

[22] M.-C. Quenez AND A. Sulem, Reflected BSDEs and robust optimal stopping for dynamic risk measures with jumps, Stochastic Process. Appl., 124 (2014), pp. 3031-3054. 